University of Wollongong

Research Online

Faculty of Engineering and Information

Faculty of Engineering and Information

Sciences - Papers: Part A

Sciences

$1-1-2016$

\title{
Fault-tolerant control of electric vehicles with in-wheel motors using actuator-grouping sliding mode controllers
}

Boyuan Li

University of Wollongong, bl995@uowmail.edu.au

Haiping Du

University of Wollongong, hdu@uow.edu.au

Weihua Li

University of Wollongong, weihuali@uow.edu.au

Follow this and additional works at: https://ro.uow.edu.au/eispapers

Part of the Engineering Commons, and the Science and Technology Studies Commons

Research Online is the open access institutional repository for the University of Wollongong. For further information contact the UOW Library: research-pubs@uow.edu.au 


\title{
Fault-tolerant control of electric vehicles with in-wheel motors using actuator- grouping sliding mode controllers
}

\begin{abstract}
Although electric vehicles with in-wheel motors have been regarded as one of the promising vehicle architectures in recent years, the probability of in-wheel motor fault is still a crucial issue due to the system complexity and large number of control actuators. In this study, a modified sliding mode control (SMC) is applied to achieve fault-tolerant control of electric vehicles with four-wheel-independent-steering (4WIS) and four-wheel-independent-driving (4WID). Unlike in traditional SMC, in this approach the steering geometry is re-arranged according to the location of faulty wheels in the modified SMC. Three SMC control laws for longitudinal velocity control, lateral velocity control and yaw rate control are designed based on specific vehicle motion scenarios. In addition the actuator-grouping SMC method is proposed so that driving actuators are grouped and each group of actuators can be used to achieve the specific control target, which avoids the strong coupling effect between each control target. Simulation results prove that the proposed modified SMC can achieve good vehicle dynamics control performance in normal driving and large steering angle turning scenarios. In addition, the proposed actuator-grouping SMC can solve the coupling effect of different control targets and the control performance is improved.
\end{abstract}

\section{Keywords}

fault, controllers, tolerant, control, electric, vehicles, wheel, motors, actuator, grouping, sliding, mode

\section{Disciplines}

Engineering | Science and Technology Studies

\section{Publication Details}

B. Li, H. Du \& W. Li, "Fault-tolerant control of electric vehicles with in-wheel motors using actuatorgrouping sliding mode controllers," Mechanical Systems and Signal Processing, vol. 72-73, pp. 462-485, 2016. 


\title{
Fault-tolerant control of electric vehicles with in-wheel motors using actuator-grouping sliding mode controllers
}

\author{
Boyuan $\mathrm{Li}^{1}$, Haiping $\mathrm{Du}^{1}$ and Weihua $\mathrm{Li}^{2}$ \\ 1. School of Electrical, Computer and Telecommunications Engineering, University of Wollongong, \\ Wollongong, NSW 2522, Australia \\ 2. School of Mechanical, Material and Mechatronic Engineering, University of Wollongong, \\ Wollongong, NSW 2522, Australia
}

\begin{abstract}
:
Although electric vehicles with in-wheel motors have been regarded as one of the promising vehicle architectures in recent years, the probability of in-wheel motor fault is still a crucial issue due to the system complexity and large number of control actuators. In this study, a modified sliding mode control (SMC) is applied to achieve fault-tolerant control of electric vehicles with four-wheel-independent-steering (4WIS) and four-wheel-independent-driving (4WID). Unlike in traditional SMC, in this approach the steering geometry is re-arranged according to the location of faulty wheels in the modified SMC. Three SMC control laws for longitudinal velocity control, lateral velocity control and yaw rate control are designed based on specific vehicle motion scenarios. In addition the actuator-grouping SMC method is proposed so that driving actuators are grouped and each group of actuators can be used to achieve the specific control target, which avoids the strong coupling effect between each control target. Simulation results prove that the proposed modified SMC can achieve good vehicle dynamics control performance in normal driving and large steering angle turning scenarios. In addition, the proposed actuator-grouping SMC can solve the coupling effect of different control targets and the control performance is improved.
\end{abstract}

Key words: yaw rate control, side-slip angle control, longitudinal velocity control, fault-tolerant control, sliding mode control, electric vehicle, in-wheel motors

\section{Introduction}

In recent years, due to the considerable potential in the reduction of emissions and fuel consumption, electric vehicles have been regarded as the promising vehicle architecture of the future. Because of the use of electric motors, such vehicles can have both four-wheelindependent-steering (4WIS) and four-wheel-independent-driving (4WID). In 4WID vehicles, four in-wheel motors are used to drive the four wheels and each individual wheel can be independently driven or controlled. Similarly, 4WIS electric vehicles can also have different 
steering angles for each wheel. Thus, for a 4WIS and 4WID electric vehicle, there is a total of eight control actuators which can be utilised to enhance the performance of traction control and direct yaw-moment control, and other advanced vehicle control strategies like energyefficient control [1]-[4].

Compared with conventional vehicles, however, the probability of an in-wheel motor fault is a crucial issue due to the system complexity and large number of control actuators. The inwheel motor fault may be caused by mechanical problems, over-heating of the motors or a fault associated with the motor drivers [5]. In addition, uneven road conditions can cause the individual wheel to lose contact with the road, thus losing friction force and this can cause a fault in an individual wheel. The fault of the in-wheel motor compromises the vehicle's dynamic control performance when conventional controllers are applied, so the design of the fault-tolerant controllers for electric vehicles is especially important.

Previously, to improve the robustness of the vehicle traction control, the model following control (MFC) approach has been proposed. This only required the input information of vehicle torque and wheel rotation speed [6-8]. Then the maximum transmissible torque estimation (MTTE) approach was developed to further improve the robust control performance of MFC [9]. Recently, a fault-tolerant control method based on MTTE has been suggested using a proportional-integral (PI) type disturbance observer [10], but this method only concerned the uncertainties of the mathematical model and sensor faults and did not focus on the failure of one specific wheel.

Driving actuator failure could be handled using the well-known $H_{\infty}$ robust control method, but the dynamic performance of the vehicle under healthy conditions was also compromised [11]. To overcome this disadvantage, various active fault-tolerant controllers (AFTC) have been proposed based on the application of a fault detection and isolation (FDI) module [12][13]. According to the fault severity, different control structures and control parameters are selected after the fault is detected. In [14], two control structures in the AFTC approach were proposed to achieve the fault-tolerant control of an induction-motor affected by a speedsensor fault. The first control structure was the PI controller for the healthy mode and the second controller was the $H_{\infty}$ robust controller for the faulty mode.

This means, however, that specific controller strategies can be implemented only after the fault has been detected and therefore fault diagnosis is important for fault-tolerant control. In the literature, a number of fault diagnosis control strategies for conventional ground vehicles have been suggested, but these control methods are not for electric vehicles [15-17]. Several fault diagnosis methods for electric vehicles have been proposed [18][19], but motor failures are hard to diagnose using only the current and voltage sensors in the in-wheel motor. In [5] and [20], the faulty wheel could be identified by estimating the individual motor control gain without the knowledge of the specific tyre-road friction coefficient.

Apart from the fault of a sensor or a fault caused by the disturbance and model uncertainty, much study has been done into the failure of the specific in-wheel motor. A control method has been proposed in which the faulty wheel and its opposite side wheel were isolated but this 
degrades the performance and stability of the vehicle [21]. Wenbo et al. proposed a control strategy to enhance the performance of the vehicle in a small turn or at low speed, but conditions where the vehicle is moving in a sharp turn or at high speed were not discussed [22]. Xin et al. classified the control strategy into the failure-driving mode, which guaranteed the vehicle continued moving and the failure-stopping mode, which stopped the vehicle [23]. In [5], a sliding mode controller (SMC) was implemented as the high level controller to achieve the desired longitudinal velocity, lateral velocity and yaw rate, then the four driving torques of each wheel could be generated to achieve these values. An adaptive-control-based passive fault-tolerant controller was also designed to maintain vehicle stability and track the desired vehicle motion [20]. Wang and Wang also introduced an improved passive faulttolerant controller which grouped the actuators having similar effects on the control of the system into one sub-system [24]. This control method was promising due to the direct distribution of the high-level control targets to each of the group of actuators in the lower level.

The adaptive control method, however, has the problem of high computational cost compared with the SMC method. For this reason many see SMC control in fault-tolerant control of 4WID vehicles as quite promising. In order to achieve better control performance, however, SMC needs large control gains and this will cause a large chattering effect. Alipour et al. suggested the proportional-integral sliding mode control (PISMC) strategy to improve the fault-tolerant control performance of the traditional SMC so that a smaller control gain could be selected and the chattering effect could be reduced [25]. Although the SMC control gain can be reduced significantly, however, the improvement of the actual dynamics control performance over the traditional SMC is not assured. Song et al. applied terminal sliding mode control (TSMC) to achieve the finite-time convergence and quick responsiveness on the terminal sliding manifold [26]. If the SMC method is applied in a 4WID vehicle to achieve multiple control targets, the control effort is allocated into the driving actuators of four wheels. One big problem is the coupling effect between different control targets and grouping the driving actuators is one of the solutions to solve this problem. For instance, the two front wheels can be considered as one group in order to control the body slip angle only, while the two rear wheels can also be regarded as one group in order to achieve the desired yaw rate. In this way, the control actuators related to the body slip angle will not have a strong effect on the control performance of the yaw rate. Except for [25] however, it appears that few researchers have examined the grouping of the driving actuators. Actuators having a similar control effect were grouped in [25], but this was not related to the coupling effect between different control targets.

This paper focuses on the fault-tolerant control method and the location of the specific faulty wheel is assumed to be known. This assumption is reasonable according to the literature [5][19][20][24]. The newly proposed SMC fault-tolerant controller focuses primarily on 4WIS-4WID electric vehicles. The main contribution of this paper is to solve the coupling effect of different control targets by grouping the actual driving actuators in fault-tolerant control of a 4WID vehicle. In addition, due to the fault of one specific wheel, the steering 
geometry of the whole vehicle will be re-arranged and the actual steering actuators will be adjusted in the 4 WIS vehicle.

The rest of this paper is organised as follows. Vehicle modelling is presented in Section 2. The steering geometry during the wheel fault is discussed in Section 3. The SMC method and its modification are shown in Section 4. The simulation results of comparing the SMC method with other stability controllers are shown in Section 5. Section 6 describes the strategy of grouping driving actuators in the SMC method to achieve better control performance. Section 7 shows the advantage of grouping the driving actuators in SMC over the traditional SMC methods. Finally, the conclusion is given in Section 8.

\section{Vehicle Dynamics Model}

\subsection{Vehicle dynamics model}

In this paper, a 4WIS-4WID vehicle model, as shown in Figure 1, is utilised to describe the dynamics motion of the electric vehicle with in-wheel steering and driving motors [27][28]. This model is used to validate the performance of the proposed control method.

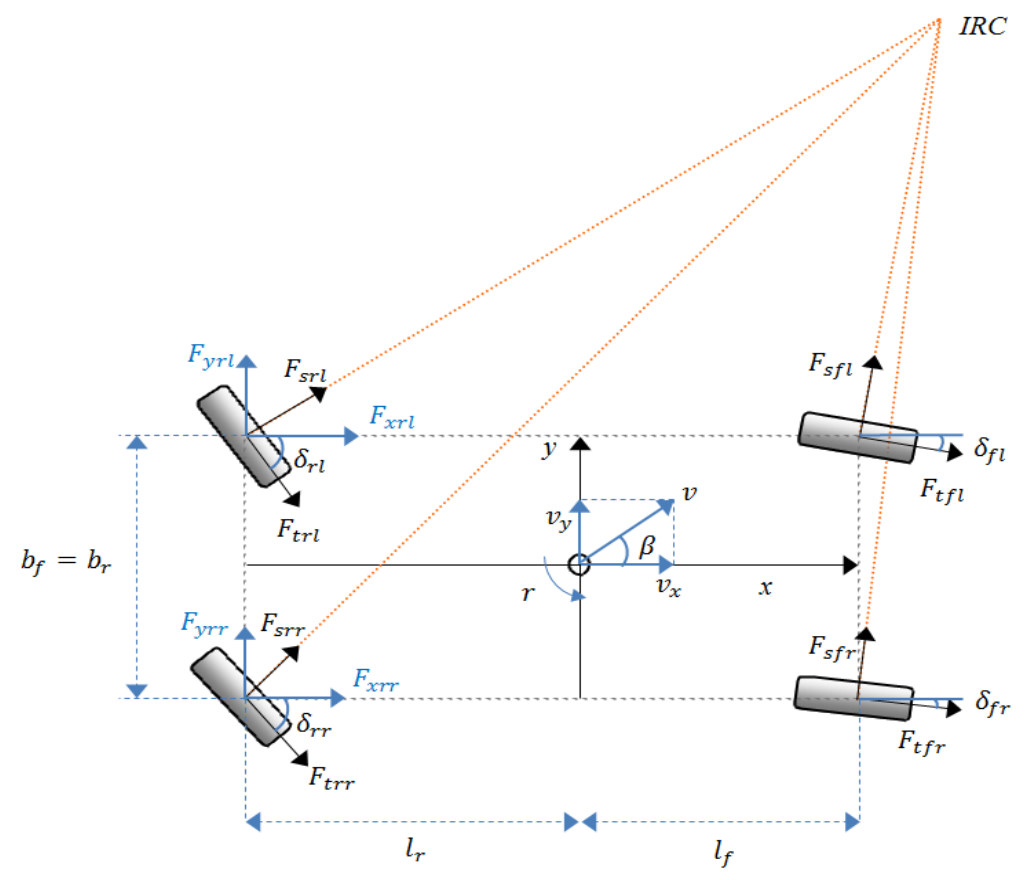

Figure 1. 4WIS-4WID vehicle dynamics model, where $I R C$ represents the instantaneous centre of rotation.

The equations of motion of this model are described as follows: 
Longitudinal motion:

$$
m \dot{v}_{x}=m v_{y} r+\left(F_{x f l}+F_{x f r}+F_{x r l}+F_{x r r}\right)
$$

Lateral motion:

$$
m \dot{v}_{y}=-m v_{x} r+\left(F_{y f l}+F_{y f r}+F_{y r l}+F_{y r r}\right)
$$

Yaw motion:

$$
I_{z} \dot{r}=l_{f}\left(F_{y f l}+F_{y f r}\right)-l_{r}\left(F_{y r l}+F_{y r r}\right)+\frac{b_{f}}{2}\left(F_{x f l}-F_{x f r}\right)+\frac{b_{r}}{2}\left(F_{x r l}-F_{x r r}\right)
$$

where $v_{x}, v_{y}, r$ are the vehicle longitudinal velocity, lateral velocity, and yaw rate, respectively. $F_{x f l}, F_{x f r}, F_{x r l}, F_{x r r}$ are the vehicle front left, front right, rear left and rear right longitudinal tyre forces, respectively, and $F_{y f l}, F_{y f r}, F_{y r l}, F_{y r r}$ are the vehicle front left, front right, rear left and rear right lateral tyre forces, respectively. $l_{f}$ and $l_{r}$ are the front and rear wheel base lengths, while $b_{f}$ and $b_{r}$ are the front and rear track widths. $I_{z}$ and $m$ are the moment of vehicle inertia in terms of yaw axis and vehicle mass.

The tyre traction or brake force and side force are defined as $F_{t i}$ and $F_{s i}$, respectively, which can be related to the longitudinal and the lateral tyre forces by the steering angle $\delta_{i}$ as follows:

$$
\begin{aligned}
& F_{x i}=F_{t i} \cos \delta_{i}-F_{s i} \sin \delta_{i} \\
& F_{y i}=F_{t i} \sin \delta_{i}+F_{s i} \cos \delta_{i}
\end{aligned}
$$

where $i=f l, f r, r l, r r$, which represents the front left, front right, rear left and rear right wheel, respectively.

\subsection{Vehicle tyre model}

The non-linear Dugoff tyre model is used in this paper [29], and is described by:

$$
\lambda_{i}=\frac{\mu F_{z i}\left[1-\varepsilon_{r} u_{i} \sqrt{s_{i}^{2}+\tan ^{2} \alpha_{i}}\right]\left(1-s_{i}\right)}{2 \sqrt{C_{s}^{2} s_{i}^{2}+C_{\alpha}^{2} \tan ^{2} \alpha_{i}}}
$$




$$
\begin{gathered}
f\left(\lambda_{i}\right)=\left\{\begin{array}{cc}
\lambda_{i}\left(2-\lambda_{i}\right) & \left(\lambda_{i}<1\right) \\
1 & \left(\lambda_{i}>1\right)
\end{array}\right. \\
F_{s i}=\frac{C_{\alpha} \tan \alpha_{i}}{1-s_{i}} f\left(\lambda_{i}\right) \\
F_{t i}=\frac{C_{s} s_{i}}{1-s_{i}} f\left(\lambda_{i}\right)
\end{gathered}
$$

where $\mu$ is the tyre-road friction coefficient. $F_{z i}$ is the vertical load of each wheel. $C_{s}$ is the longitudinal cornering stiffness and $C_{\alpha}$ is the lateral cornering stiffness. $s_{i}$ is the longitudinal slip ratio, and $\alpha_{i}$ is the lateral slip angle. $\varepsilon_{r}$ is a constant value, and $v_{L i}$ is the vehicle velocity component in the wheel plane which is defined for each wheel as:

$$
\begin{aligned}
& v_{L f l}=\left(v_{x}+\frac{1}{2} b_{f} r\right) \cos \delta_{f l}+\left(v_{y}+l_{f} r\right) \sin \delta_{f l} \\
& v_{L f r}=\left(v_{x}-\frac{1}{2} b_{f} r\right) \cos \delta_{f r}+\left(v_{y}+l_{f} r\right) \sin \delta_{f r} \\
& v_{L r l}=\left(v_{x}+\frac{1}{2} b_{r} r\right) \cos \delta_{r l}-\left(l_{r} r-v_{y}\right) \sin \delta_{r l} \\
& v_{L r r}=\left(v_{x}-\frac{1}{2} b_{r} r\right) \cos \delta_{r r}-\left(l_{r} r-v_{y}\right) \sin \delta_{r r}
\end{aligned}
$$

\section{Steering Geometry while Wheel-fault Happening}

According to [30][31], a complete steering model for an individual wheel of the electric vehicle can be presented by the following equation:

$$
J_{e f f} \ddot{\delta}_{i}+b_{e f f} \dot{\delta}_{i}+k \delta_{i}=\tau_{a}+\tau_{j}+\tau_{a c t}
$$

where $J_{\text {eff }}$ is the effective moment of inertia and $b_{\text {eff }}$ is the effective damping coefficient. $k$ is the jack-up moment coefficient. $\tau_{a}$ is the total alignment moment, which is the moment generated about the steering axis from the lateral force. $\tau_{j}$ is the reaction torque produced from the vertical tyre force and suspension travel as a function of steering angle. $\tau_{\text {act }}$ is the actual steering torque generated from the steering motor. The output steering angle $\delta_{i}$ can be controlled by adjusting the actual steering torque $\tau_{\text {act }}$ according to the desired steering angle given by the driver. Therefore, the steering angle of an individual wheel $\delta_{i}$ is assumed to be known when all the wheels work well. However, when an individual steering motor cannot 
work, the actual steering torque is zero and the output steering angle is governed by the following equation:

$$
J_{e f f} \ddot{\delta}_{i}+b_{e f f} \dot{\delta}_{i}+k \delta_{i}=\tau_{a}+\tau_{j}
$$

In [32], it is suggested that the jacking torque does not play the important role when the vehicle longitudinal velocity is large and the tyre lateral force is large. In this study, the vehicle initial longitudinal velocity is $20 \mathrm{~m} / \mathrm{s}$ or $15 \mathrm{~m} / \mathrm{s}$, which is quite large. Thus, the effect of jacking torque can be neglected and the steering angle is determined by the total alignment moment $\tau_{a}$ :

$$
\tau_{a}=-\left(t_{m}+t_{p 0}-\frac{t_{p 0} C_{\alpha}}{3 \mu F_{z i}}\left|\tan \alpha_{i}\right|\right) F_{s i}
$$

where $t_{m}$ is the mechanical trail and $t_{p 0}$ is the initial pneumatic trail.

If one wheel is faulty during vehicle turning, the steering angle of other three wheels must be adjusted according to the steering geometry in Figure 2 to maintain the turning. One simple method to realise the geometry in Figure 2 is shown as follows:

1) If the faulty wheel is the front wheel, the vehicle $I C R$ is located on the extension cord of the front track. The steering angle of the healthy front wheel is zero and the steering angle of the front faulty wheel is determined by equations (8) and (9). The steering angles of the rear left and rear right wheels can be calculated as [33]:

$$
\delta_{i}=\tan ^{-1}\left(D^{T}\left(x_{i}-x_{I C R}\right),-D^{T}\left(y_{i}-y_{I C R}\right)\right)
$$

where $x_{I C R}=l_{f}$ and $y_{I C R}=\frac{l_{f}+l_{r}}{\delta_{d}} . i=r l, r r$, which presents the rear left and rear right wheel, respectively. $\delta_{d}$ is the desired steering angle, which is determined by the driver. $\left(x_{r l}, y_{r l}\right)=$ $\left(-l_{r}, \frac{b_{f}}{2}\right)$ and $\left(x_{r r}, y_{r r}\right)=\left(-l_{r},-\frac{b_{f}}{2}\right)$ are the positions of the wheel centre. $D^{T}=1$, if turning in an anti-clockwise direction; $D^{T}=-1$, if turning in a clockwise direction.

It should be noted that if the driver's desired steering centre is $\delta_{d}$, the vehicle turning radius is $R=\frac{l_{f}+l_{r}}{\delta_{d}}$. The turning radius is the distance between the vehicle centre of gravity and the $I C R$ but in this paper it is assumed that the distance between the front wheel centre (rear wheel centre) and the ICR is the turning radius. This assumption is reasonable because usually, the turn radius is much larger than the vehicle wheel base.

2) If the faulty wheel is the rear wheel, the vehicle $I C R$ is located on the extension cord of the rear track. The steering angle of the rear healthy wheel is zero and the steering angle of the rear faulty wheel can be determined by equations (8) and (9). The steering angles of the front left and front right wheel can be calculated using equation (10). In equation (10), $i=f l, f r$, which represents the front left and front right wheel, respectively. In addition, $x_{I C R}=-l_{r}$ and $y_{I C R}=\frac{l_{f}+l_{r}}{\delta_{d}} .\left(x_{r l}, y_{r l}\right)=\left(-l_{r}, \frac{b_{f}}{2}\right)$ and $\left(x_{r r}, y_{r r}\right)=\left(-l_{r},-\frac{b_{f}}{2}\right)$ are positions of the wheel centre. $D^{T}=1$, if the turning is anti-clockwise; $D^{T}=-1$, if the turning is clockwise. 
The motor driver and in-wheel motor driving unit can be described by a control gain $k_{i}$, which is related to the in-wheel driving motor of each wheel.

$$
k_{i}=\frac{T_{i}}{u_{i}}
$$

where $T_{i}$ is the driving torque of each wheel of the in-wheel motor and $u_{i}$ is the input voltage of the in-wheel motor.

If the driving wheel is in a healthy condition, the normalised control gain $k_{i}$ is assumed to be equal to 1 . The value of $k_{i}$ can be estimated by various fault diagnosis methods. [5][20] The specific faulty wheel can be identified according to the estimation results of the control gain of driving torque $k_{i}$.
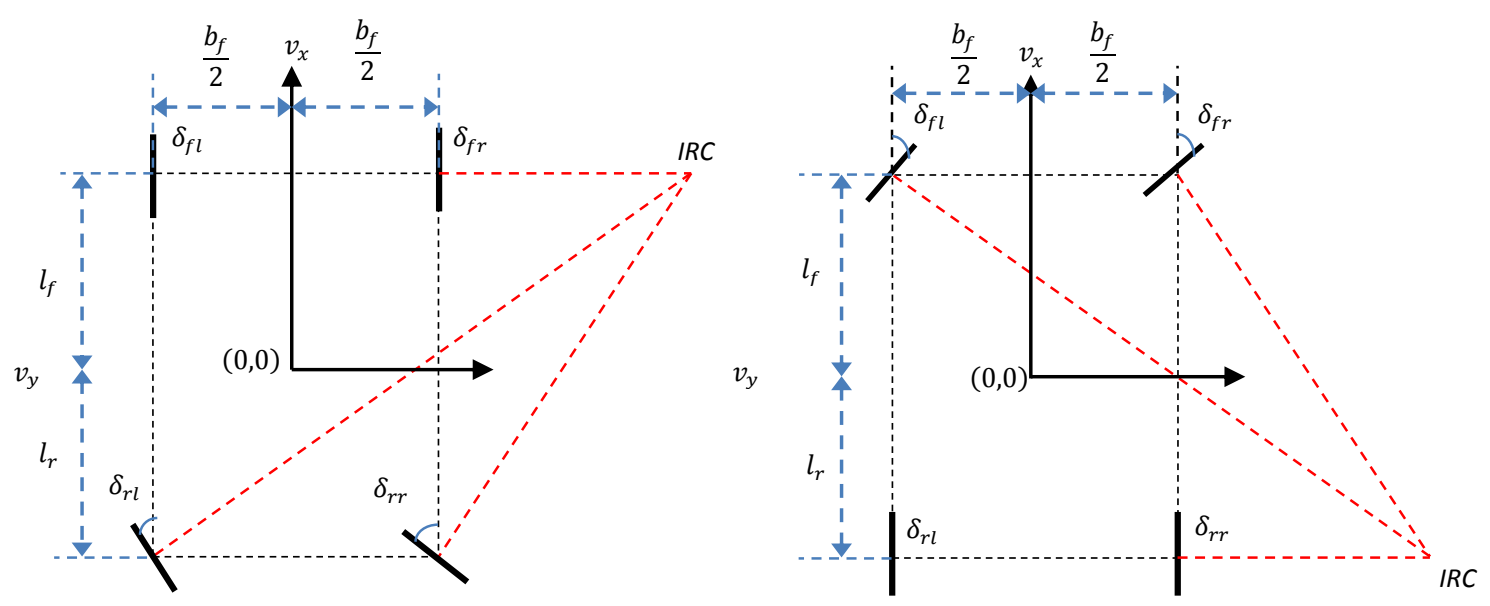

Figure 2. The vehicle steering geometry when one of the four wheels is the faulty wheel.

It should be noted that if the two front wheels of the vehicle cannot work during turning, the vehicle can move like a rear wheel steering vehicle. Similarly, if the two rear wheels of the vehicle are faulty during turning, the vehicle can move as a front wheel steering vehicle. However, if one of the front wheels and one of the rear wheels cannot work or more than three wheels are faulty, the vehicle cannot make a turn and must stop.

\section{Sliding Mode Controller Design}

To evaluate the SMC, the vehicle dynamics equation (1)-(3) can be simplified using the following equations [5]:

$$
\begin{aligned}
\dot{v}_{x} & =v_{y} r+\frac{1}{m} F_{x} \\
\dot{v}_{y} & =-v_{x} r+\frac{1}{m} F_{y} \\
\dot{r} & =\frac{1}{I_{z}} M_{z}
\end{aligned}
$$


where $F_{x}, F_{y}$ and $M_{z}$ are the total longitudinal force, lateral force and yaw moment.

$$
\begin{gathered}
F_{x}=F_{x f l}+F_{x f r}+F_{x r l}+F_{x r r} \\
F_{y}=F_{y f l}+F_{y f r}+F_{y r l}+F_{y r r} \\
M_{z}=l_{f}\left(F_{y f l}+F_{y f r}\right)-l_{r}\left(F_{y r l}+F_{y r r}\right)+\frac{b_{f}}{2}\left(F_{x f l}-F_{x f r}\right)+\frac{b_{r}}{2}\left(F_{x r l}-F_{x r r}\right)
\end{gathered}
$$

According to [20], equation (12) can be rewritten as:

$$
\left[\begin{array}{c}
\dot{v}_{x} \\
\dot{v}_{y} \\
\dot{r}
\end{array}\right]=\left[\begin{array}{c}
v_{y} r \\
-v_{x} r \\
0
\end{array}\right]+B_{y}\left(\delta_{i}\right) \boldsymbol{F}_{\boldsymbol{s}}+B_{x}\left(\delta_{i}\right) \boldsymbol{F}_{\boldsymbol{t}}
$$

where $\boldsymbol{F}_{\boldsymbol{t}}=\left[\begin{array}{llllll}F_{t f l} & F_{t f r} & F_{t r l} & F_{t r r}\end{array}\right]^{\boldsymbol{T}}, \boldsymbol{F}_{\boldsymbol{s}}=\left[\begin{array}{lllll}F_{s f l} & F_{s f r} & F_{s r l} & F_{s r r}\end{array}\right]^{\boldsymbol{T}}$, which presents the tyre force along the wheel direction and perpendicular to the wheel direction, respectively.

$$
\begin{aligned}
& B_{x}\left(\delta_{i}\right) \\
& =\left[\begin{array}{ccc}
\frac{1}{m} & 0 & 0 \\
0 & \frac{1}{m} & 0 \\
0 & 0 & \frac{1}{I_{z}}
\end{array}\right]\left[\begin{array}{rlcc}
\cos \delta_{f l} & \cos \delta_{f r} & \cos \delta_{r l} & \cos \delta_{r r} \\
\sin \delta_{f l} & \sin \delta_{f r} & \sin \delta_{r l} & \sin \delta_{r r} \\
l_{f} \sin \delta_{f l}+\frac{b_{f}}{2} \cos \delta_{f l} & l_{f} \sin \delta_{f r}-\frac{b_{f}}{2} \cos \delta_{f r} & \frac{b_{r}}{2} \cos \delta_{r l}-l_{r} \sin \delta_{r l} & -\frac{b_{r}}{2} \cos \delta_{r r}-l_{r} \sin \delta_{r r}
\end{array}\right] \\
& B_{y}\left(\delta_{i}\right) \\
& =\left[\begin{array}{ccc}
\frac{1}{m} & 0 & 0 \\
0 & \frac{1}{m} & 0 \\
0 & 0 & \frac{1}{I_{z}}
\end{array}\right]\left[\begin{array}{rlcc}
-\sin \delta_{f l} & -\sin \delta_{f r} & -\sin \delta_{r l} & -\sin \delta_{r r} \\
\cos \delta_{f l} & \cos \delta_{f r} & \cos \delta_{r l} & \cos \delta_{r r} \\
l_{f} \cos \delta_{f l}-\frac{b_{f}}{2} \sin \delta_{f l} & l_{f} \cos \delta_{f r}+\frac{b_{f}}{2} \sin \delta_{f r} & -\frac{b_{r}}{2} \sin \delta_{r l}-l_{r} \cos \delta_{r l} & \frac{b_{r}}{2} \sin \delta_{r r}-l_{r} \cos \delta_{r r}
\end{array}\right]
\end{aligned}
$$

According to the wheel dynamics and equation (11), the individual tyre longitudinal force $F_{t i}$ can be written as:

$$
F_{t i}=\frac{k_{i} u_{i}-I_{\omega} \dot{\omega}_{i}}{R_{\omega}}
$$

Therefore, the longitudinal tyre force can be presented by the following equation:

$$
\boldsymbol{F}_{\boldsymbol{t}}=\frac{1}{R_{\omega}}\left[\begin{array}{cccc}
k_{f l} & 0 & 0 & 0 \\
0 & k_{f r} & 0 & 0 \\
0 & 0 & k_{r l} & 0 \\
0 & 0 & 0 & k_{r r}
\end{array}\right]\left[\begin{array}{c}
u_{f l} \\
u_{f r} \\
u_{r l} \\
u_{r r}
\end{array}\right]-\frac{I_{\omega}}{R_{\omega}}\left[\begin{array}{c}
\dot{\omega}_{f l} \\
\dot{\omega}_{f r} \\
\dot{\omega}_{r l} \\
\dot{\omega}_{r r}
\end{array}\right]
$$

Based on (16), vehicle model (14) can be rewritten as: 


$$
\left[\begin{array}{c}
\dot{v}_{x} \\
\dot{v}_{y} \\
\dot{r}
\end{array}\right]=\left[\begin{array}{c}
v_{y} r \\
-v_{x} r \\
0
\end{array}\right]+B_{y}\left(\delta_{i}\right) \boldsymbol{F}_{s}+\frac{B_{x}\left(\delta_{i}\right)}{R_{\omega}}\left(\left[\begin{array}{cccc}
k_{f l} & 0 & 0 & 0 \\
0 & k_{f r} & 0 & 0 \\
0 & 0 & k_{r l} & 0 \\
0 & 0 & 0 & k_{r r}
\end{array}\right]\left[\begin{array}{c}
u_{f l} \\
u_{f r} \\
u_{r l} \\
u_{r r}
\end{array}\right]-I_{\omega}\left[\begin{array}{c}
\dot{\omega}_{f l} \\
\dot{\omega}_{f r} \\
\dot{\omega}_{r l} \\
\dot{\omega}_{r r}
\end{array}\right]\right)
$$

It is assumed that the lateral tyre force can be described by the following equation related to the side-slip angle if the linear relationship is assumed:

$$
F_{\text {si-linear }}=C_{a} \alpha_{i}\left(v_{x}, v_{y}, r\right)
$$

If the non-linear tyre characteristic is considered, the tyre lateral force can be presented as follows:

$$
F_{s i}=F_{\text {si-linear }}+\Delta F_{s i}=C_{a} \alpha_{i}\left(v_{x}, v_{y}, r\right)+\Delta F_{s i}
$$

$\Delta F_{s i}$ represents the additional lateral tyre force caused by the non-linear tyre characteristic.

In this way, equation (17) can be rewritten as equation (20) by neglecting the lateral tyre nonlinear characteristics and acceleration of the wheel angular velocity. This simplification is reasonable because these neglected values can be compensated for by increasing the sliding mode gain.

$$
\begin{aligned}
& {\left[\begin{array}{c}
\dot{v}_{x} \\
\dot{v}_{y} \\
\dot{r}
\end{array}\right]=\left[\begin{array}{c}
v_{y} r \\
-v_{x} r \\
0
\end{array}\right]+B_{y}\left(\delta_{i}\right) \boldsymbol{F}_{\boldsymbol{s}-\text { linear }}+\frac{1}{R_{\omega}}\left[\begin{array}{ccc}
\frac{1}{m} & 0 & 0 \\
0 & \frac{1}{m} & 0 \\
0 & 0 & \frac{1}{I_{z}}
\end{array}\right]} \\
& {\left[\begin{array}{clll}
\cos \delta_{f l} k_{f l} & \cos \delta_{f r} k_{f r} & \cos \delta_{r l} k_{r l} & \cos \delta_{r r} k_{r r} \\
\sin \delta_{f l} k_{f l} & \sin \delta_{f r} k_{f r} & \sin \delta_{r l} k_{r l} & \sin \delta_{r r} k_{r r} \\
\left(l_{f} \sin \delta_{f l}+\frac{b_{f}}{2} \cos \delta_{f l}\right) k_{f l} & \left(l_{f} \sin \delta_{f r}-\frac{b_{f}}{2} \cos \delta_{f r}\right) k_{f r} & \left(\frac{b_{r}}{2} \cos \delta_{r l}-l_{r} \sin \delta_{r l}\right) k_{r l} & \left(-\frac{b_{r}}{2} \cos \delta_{r r}-l_{r} \sin \delta_{r r}\right) k_{r r}
\end{array}\right]} \\
& {\left[\begin{array}{l}
u_{f l} \\
u_{f r} \\
u_{r l} \\
u_{r r}
\end{array}\right]}
\end{aligned}
$$

where $\boldsymbol{F}_{\text {s-linear }}=\left[\begin{array}{l}C_{a} \alpha_{f l}\left(v_{x}, v_{y}, r\right) \\ C_{a} \alpha_{f r}\left(v_{x}, v_{y}, r\right) \\ C_{a} \alpha_{r l}\left(v_{x}, v_{y}, r\right) \\ C_{a} \alpha_{r r}\left(v_{x}, v_{y}, r\right)\end{array}\right]$

The SMC control law is evaluated according to equation (20):

$$
\dot{v}_{x}=v_{y} r+B_{y 1} \boldsymbol{F}_{\text {s-linear }}+v_{1}
$$




$$
\begin{gathered}
\dot{v}_{y}=-v_{x} r+B_{y 2} \boldsymbol{F}_{\text {s-linear }}+v_{2} \\
\dot{r}=B_{y 3} \boldsymbol{F}_{\text {s-linear }}+v_{3}
\end{gathered}
$$

where $B_{y 1}=\left[\begin{array}{llll}-\frac{\sin \delta_{f l}}{m} & -\frac{\sin \delta_{f r}}{m} & -\frac{\sin \delta_{r l}}{m} & -\frac{\sin \delta_{r r}}{m}\end{array}\right], B_{y 2}=\left[\begin{array}{llll}\frac{\cos \delta_{f l}}{m} & \frac{\cos \delta_{f r}}{m} & \frac{\cos \delta_{r l}}{m} & \frac{\cos \delta_{r r}}{m}\end{array}\right], B_{y 3}=$ $\left[\frac{1}{I_{z}}\left(l_{f} \cos \delta_{f l}-\frac{b_{f}}{2} \sin \delta_{f l}\right) \quad \frac{1}{I_{z}}\left(l_{f} \cos \delta_{f r}+\frac{b_{f}}{2} \sin \delta_{f r}\right) \quad \frac{1}{I_{z}}\left(-\frac{b_{r}}{2} \sin \delta_{r l}-l_{r} \cos \delta_{r l}\right) \quad \frac{1}{I_{z}}\left(\frac{b_{r}}{2} \sin \delta_{r r}-l_{r} \cos \delta_{r r}\right)\right]$

The control law can be chosen such as:

$$
\begin{gathered}
v_{1}=-v_{y} r-B_{y 1} \boldsymbol{F}_{\text {s-linear }}+\dot{v}_{x r}-K_{1} \operatorname{sgn}\left(S_{1}\right) \\
v_{2}=v_{x} r-B_{y 2} \boldsymbol{F}_{\text {s-linear }}+\dot{v}_{y r}-K_{2} \operatorname{sgn}\left(S_{2}\right) \\
v_{3}=-B_{y 3} \boldsymbol{F}_{\text {s-linear }}+\dot{r}_{r}-K_{3} \operatorname{sgn}\left(S_{3}\right)
\end{gathered}
$$

where $v_{x r}, v_{y r}$ and $r_{r}$ present the desired longitudinal velocity, lateral velocity and yaw rate. $\dot{v}_{x r}, \dot{v}_{y r}$ and $\dot{r}_{r}$ and the desired longitudinal acceleration, lateral acceleration and yaw acceleration. $K_{1}, K_{2}$ and $K_{3}$ are the control gains of SMC corresponding to $v_{1}, v_{2}$ and $v_{3}$ respectively.

The sliding surface of each channel $S_{1}, S_{2}$ and $S_{3}$ can be defined as:

$$
S_{n}=x_{n}-x_{n r}
$$

where $n=1,2,3, x_{n r}$ is the vehicle state reference $\left(v_{x r}, v_{y r}, r_{r}\right)$ and $x_{n}$ is the vehicle state $\left(v_{x}, v_{y}, r\right)$.

To prove the stability of the suggested control law, the Lyapunov method is used. The Lyapunov functions for the three channels can be chosen as:

$$
V_{n}=\frac{1}{2} S_{n}^{2}
$$

The time derivative of the above Lyapunov function is:

$$
\begin{gathered}
\dot{V}_{1}=S_{1} \dot{S}_{1}=S_{1}\left(\dot{v}_{x}-\dot{v}_{x r}\right)=S_{1}\left(v_{y} r+B_{y 1} \boldsymbol{F}_{\text {s-linear }}+v_{1}-\dot{v}_{x r}\right) \\
\dot{V}_{2}=S_{2} \dot{S}_{2}=S_{2}\left(\dot{v}_{y}-\dot{v}_{y r}\right)=S_{2}\left(-v_{x} r+B_{y 2} \boldsymbol{F}_{\text {s-linear }}+v_{2}-\dot{v}_{y r}\right) \\
\dot{V}_{3}=S_{3} \dot{S}_{3}=S_{3}\left(\dot{r}-\dot{r}_{r}\right)=S_{3}\left(B_{y 3} \boldsymbol{F}_{\text {s-linear }}+v_{3}-\dot{r}_{r}\right)
\end{gathered}
$$

By applying the suggested control law in equation (22), equation (25) is rewritten as:

$$
\begin{aligned}
& \dot{V}_{1}=S_{1} \dot{S}_{1}=-S_{1} K_{1} \operatorname{sgn}\left(S_{1}\right)=-K_{1}\left|S_{1}\right| \\
& \dot{V}_{2}=S_{2} \dot{S}_{2}=-S_{2} K_{2} \operatorname{sgn}\left(S_{2}\right)=-K_{2}\left|S_{2}\right| \\
& \dot{V}_{3}=S_{3} \dot{S}_{3}=-S_{3} K_{3} \operatorname{sgn}\left(S_{3}\right)=-K_{3}\left|S_{3}\right|
\end{aligned}
$$


According to equation (26), the time derivative of the above Lyapunov function is always negative, which proves the stability of the whole system.

To achieve the control law in equation (22), the actual driving torque of each wheel should be distributed according to equation (21):

$$
\begin{gathered}
v_{1}=\frac{1}{R_{\omega} m}\left(\cos \delta_{f l} k_{f l} u_{f l}+\cos \delta_{f r} k_{f r} u_{f r}+\cos \delta_{r l} k_{r l} u_{r l}+\cos \delta_{r r} k_{r r} u_{r r}\right)=-v_{y} r- \\
B_{y 1} \boldsymbol{F}_{s-\text { linear }}+\dot{v}_{x r}-K_{1} \operatorname{sgn}\left(S_{1}\right) \\
v_{2}=\frac{1}{R_{\omega} m}\left(\sin \delta_{f l} k_{f l} u_{f l}+\sin \delta_{f r} k_{f r} u_{f r}+\sin \delta_{r l} k_{r l} u_{r l}+\sin \delta_{r r} k_{r r} u_{r r}\right)=v_{x} r- \\
B_{y 2} \boldsymbol{F}_{s-\text { linear }}+\dot{v}_{y r}-K_{2} \operatorname{sgn}\left(S_{2}\right) \\
v_{3}=\frac{1}{R_{\omega} I_{z}}\left(\left(l_{f} \sin \delta_{f l}+\frac{b_{f}}{2} \cos \delta_{f l}\right) k_{f l} u_{f l}+\left(l_{f} \sin \delta_{f r}-\frac{b_{f}}{2} \cos \delta_{f r}\right) k_{f r} u_{f r}+\left(\frac{b_{r}}{2} \cos \delta_{r l}-\right.\right. \\
\left.\left.l_{r} \sin \delta_{r l}\right) k_{r l} u_{r l}+\left(-\frac{b_{r}}{2} \cos \delta_{r r}-l_{r} \sin \delta_{r r}\right) k_{r r} u_{r r}\right)=-B_{y 3} \boldsymbol{F}_{s-\text { linear }}+\dot{r}_{r}-K_{3} \operatorname{sgn}\left(S_{3}\right)
\end{gathered}
$$

It is noted that the longitudinal velocity, lateral velocity and yaw rate are assumed to be available. Lateral tyre force $\boldsymbol{F}_{\boldsymbol{s} \text {-linear }}$ can be estimated according to the linear tyre model and the side-slip angle of each individual wheel and the non-linear tyre characteristic is neglected. These assumptions are reasonable because the estimation of the longitudinal velocity, lateral velocity, longitudinal slip ratio, friction coefficient and lateral slip angle of the vehicle has been done previously. [34-38] The actual vehicle yaw rate $r$ can be measured by inertial measurement unit (IMU).

The function $\operatorname{sgn}(x)$ used in SMC control law (27) will cause the serious chattering effect due to the abrupt change. In order to achieve continues and smooth switching control law, the saturation function $\operatorname{Sat}(x)$ is used as follows instead of $\operatorname{sgn}(x)$ in SMC:

$$
\operatorname{Sat}(x)=\left\{\begin{array}{cc}
1 & \text { if } x>\varepsilon \\
\operatorname{sgn}(x) & \text { if }-\varepsilon \leq x \leq \varepsilon \\
-1 & \text { if } x<-\varepsilon
\end{array}\right.
$$

where $\varepsilon$ is the thickness of the boundary layer.

The desired longitudinal acceleration $\dot{v}_{x r}$ is determined by driver's desired driving input $T_{d}$, which is shown in equation (29). The desired longitudinal velocity $v_{x r}$ can be determined by the integration of the desired longitudinal acceleration.

$$
\dot{v}_{x r}=\frac{T_{d}}{m R_{\omega}}
$$


The desired lateral velocity and lateral acceleration are assumed as zero. The lateral velocity is related to the vehicle body slip angle, which is an important value to present the vehicle stability. Boada et al. suggested the vehicle desired body slip angle is zero [28]:

$$
\beta_{d}=0
$$

The desired yaw rate can be calculated by the following equation [28]:

$$
r_{r}=\frac{v_{x 0} \delta_{d}}{\left(l_{f}+l_{r}\right)\left(1+P v_{x 0}^{2}\right)}
$$

where $P=-\frac{m}{2\left(l_{f}+l_{r}\right)^{2}} \frac{C_{\alpha f}\left(l_{f}-l_{r}\right)}{C_{a f} C_{a r}}$, which is defined as the stability factor. $C_{\alpha f}$ and $C_{\alpha r}$ are the front tyre and rear tyre cornering stiffness, which are assumed as the same value $C_{\alpha}$ in this research. $v_{x 0}$ is the initial vehicle longitudinal velocity. The desired yaw acceleration is determined by the derivative of the desired yaw rate.

When a particular driving motor fails, the corresponding driving gain $k_{i}$ can be reduced to reflect this failure. The vehicle handling and stability can be guaranteed by achieving the virtual control values $v_{1}, v_{2}$ and $v_{3}$ in SMC.

In the traditional SMC method, the distributed input voltage $u_{i}$ of each driving motor can be solved by equation (27). However, there are four variables in three equations. Even if one of the driving motors is faulty, we actually do not need to control all these three control targets under certain scenarios. For example, only control of the longitudinal velocity and yaw rate are required under normal driving conditions with a small steering angle. If the vehicle is turning with a large steering angle, only control of the yaw rate and body slip angle (related to the lateral velocity) is required.

Therefore, the SMC control system in equation (27) can be considered as an over-actuated control allocation problem. An allocation optimisation method can be proposed to minimise the control effort of each individual driving motor and meet the constraints of equation (27) at the same time. The constraints (27a), (27b) and (27c) can be chosen according to the actual scenarios. The cost function of the optimisation problem is:

$$
J=\frac{1}{k_{f l}} u_{f l}^{2}+\frac{1}{k_{f r}} u_{f r}^{2}+\frac{1}{k_{r l}} u_{r l}^{2}+\frac{1}{k_{r r}} u_{r r}^{2}
$$

s.t. equations (27a), (27b) and (27c).

The cost function (32) can minimise the driving effort of individual wheels. Meanwhile, including the motor driving gain can counterbalance the large driving effort of a faulty wheel.

In addition, the practical limitation of the the maximum driving torque of the individual wheel for the electric vehicle $T_{\max }$ is 250 N.m [25]. Thus, the additional constraint of the control input is added into (32): 


$$
-T_{\max } \leq k_{i} u_{i} \leq T_{\max }
$$

The allocated driving torque should also consider the effect of tyre force saturation. The actual total longitudinal tyre force and lateral tyre force generated by a specific tyre are limited by the vertical load of the wheel and the tyre-road friction coefficient. The following friction circle constraint is widely used in to describe the tyre force saturation:

$$
F_{t i}^{2}+F_{s i}^{2} \leq \mu F_{z i}^{2}
$$

However, this nonlinear constraint will greatly increase the computational effort. Castro et al. suggested this nonlinear inequality can be approximated by the $\mathrm{N}$ half-spaces: [39]

$$
C\left[\begin{array}{l}
F_{t i} \\
F_{s i}
\end{array}\right] \leq D \mu F_{z i}
$$

where $C \in R^{N \times 2}, D \in R^{N}$ are matrices that characterise the half-spaces. One may see that, as the number of half-space $N$ is increased, the friction circle constraints can be approximated with increasing accuracy. However, during the actual implementation of the controller, $N$ cannot be too large, as the computational effort will greatly increase. To improve the computational efficiency, $F_{t i}$ can be calculated from the driving torque (equation (36)) and $F_{s i}$ is equal to $\boldsymbol{F}_{\boldsymbol{s} \text {-linear }}$.

$$
F_{t i}=\frac{k_{i} u_{i}}{R_{\omega}}
$$

The inequality (35) can be considered as another constraint of the optimisation problem (32).

V. Numerical Comparison between SMC Controller and other Vehicle Dynamics Controllers

In this section, two sets of simulations are used to test the proposed SMC fault-tolerant controller. These simulation results are also compared with the traditional vehicle dynamics controller which does not consider the fault-tolerant problem. The traditional method uses the linear feedback method to adjust the four steering angles and four driving torques in order to achieve the desired yaw rate and body slip angle. We denote this as the linear feedback method in the following paragraph [32]. In addition, the simulation results during the situation where no controller is applied are also presented in order to verify the SMC control performance. The vehicle parameters used in the simulations are listed in Table 1.

In the first set of simulations, the motion of a single lane change is examined. The driver's input steering angle is shown in Figure 3 and the friction coefficient is assumed as 0.9. The vehicle initial velocity is $20 \mathrm{~m} / \mathrm{s}$. It is assumed that the wheel fault first happens in the rear right wheel from 2 seconds to 2.5 seconds. The driver still wants to accelerate the vehicle and 
the desired total driving torque is shown in Figure 4. In the proposed SMC controller, only the longitudinal velocity control law $v_{1}$ and yaw rate control law $v_{3}$ are applied here. This is because the longitudinal velocity and yaw rate are primary control targets under normal driving conditions. The value of the allocated driving torque is determined by the control law $v_{1}$ to overcome the friction force and achieve the desired longitudinal velocity. In the linear feedback controller and no controller applied conditions, the desired driving torque in Figure 4 is equally distributed to two rear wheels.

Figure 5 suggests that the proposed SMC method and the linear feedback controller can both achieve the desired yaw rate accurately compared with when there is no controller applied. The SMC method shows robust control performance when the rear right wheel is faulty over a period of time. The linear feedback controller uses the driving torque to adjust the yaw rate. Under normal driving conditions, this control effort is small and the loss of working effort of one wheel will not significantly impair the yaw rate control performance. Figure 6 shows that the proposed SMC method can better achieve the desired longitudinal velocity compared with the linear feedback controller due to the applied control law $v_{1}$ (channel 1 of SMC).

Next, the wheel fault is assumed to occur in the two rear wheels from 2 seconds to 2.5 seconds. Figure 7 and Figure 8 also compare the control performance of yaw rate and longitudinal velocity between the proposed SMC method and the use of a linear feedback controller. The linear feedback controller has a serious error at 2.5 seconds and the simulation stops because the scenario that both the two rear wheels are faulty is more challenging than one faulty wheel scenario. The proposed SMC method can achieve the desired yaw rate even if the two rear wheels cannot work properly from 2 seconds to 2.5 seconds. The desired longitudinal velocity can be also better achieved due to the applied control law $v_{1}$.

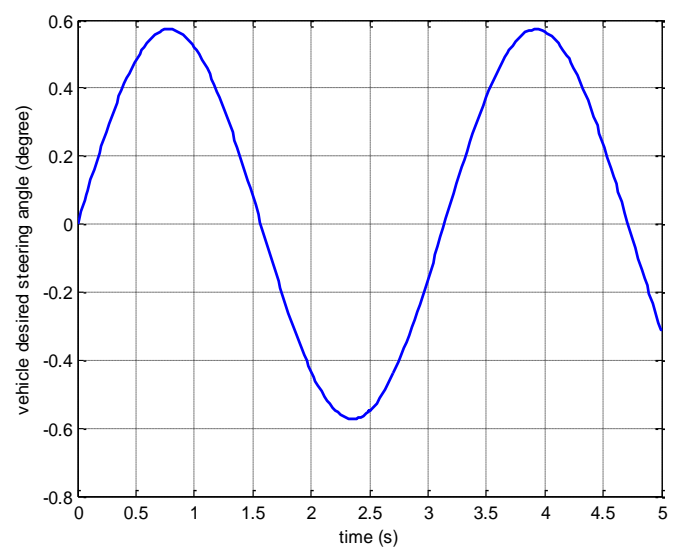

Figure 3. Driver's steering input during the motion of single lane change. 


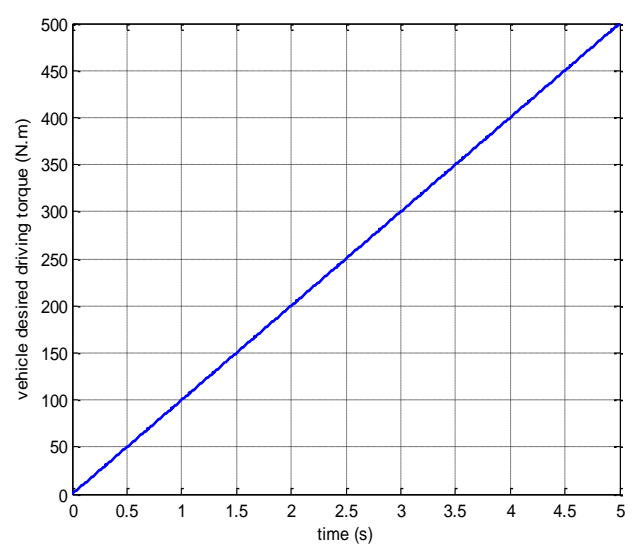

Figure 4. Driver's desired driving input during the motion of single lane change.
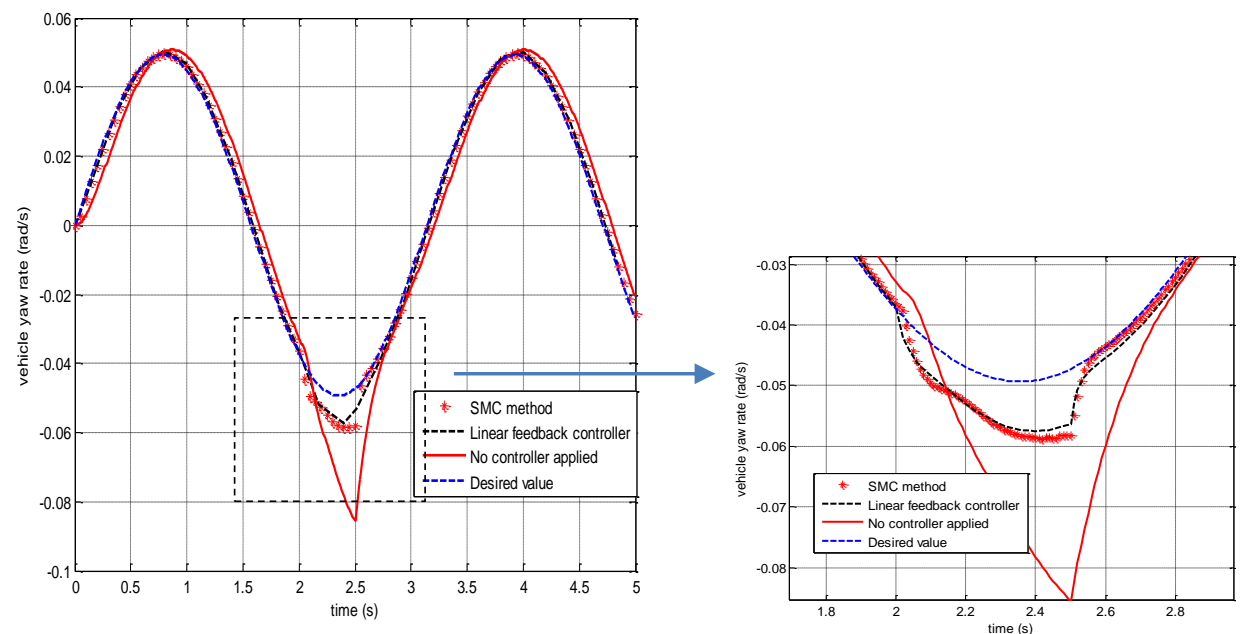

Figure 5. Vehicle controlled yaw rate during the motion of single lane change. (one faulty wheel)

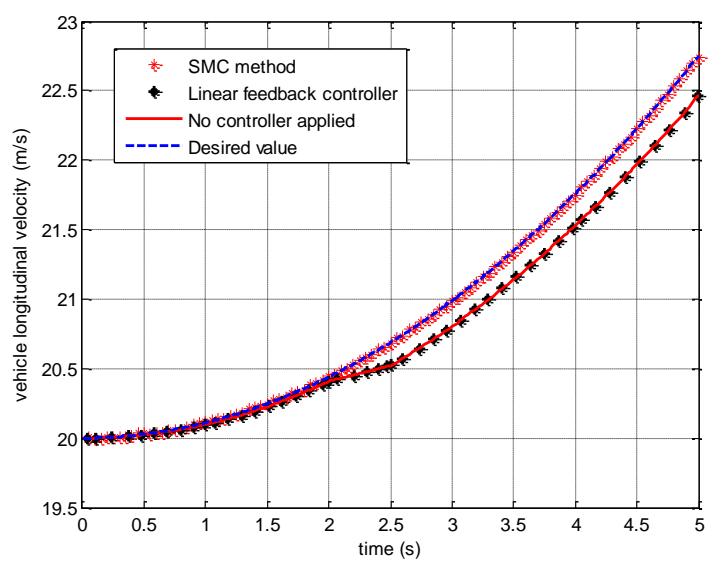

Figure 6. Vehicle longitudinal velocity during the motion of single lane change. (one faulty wheel) 

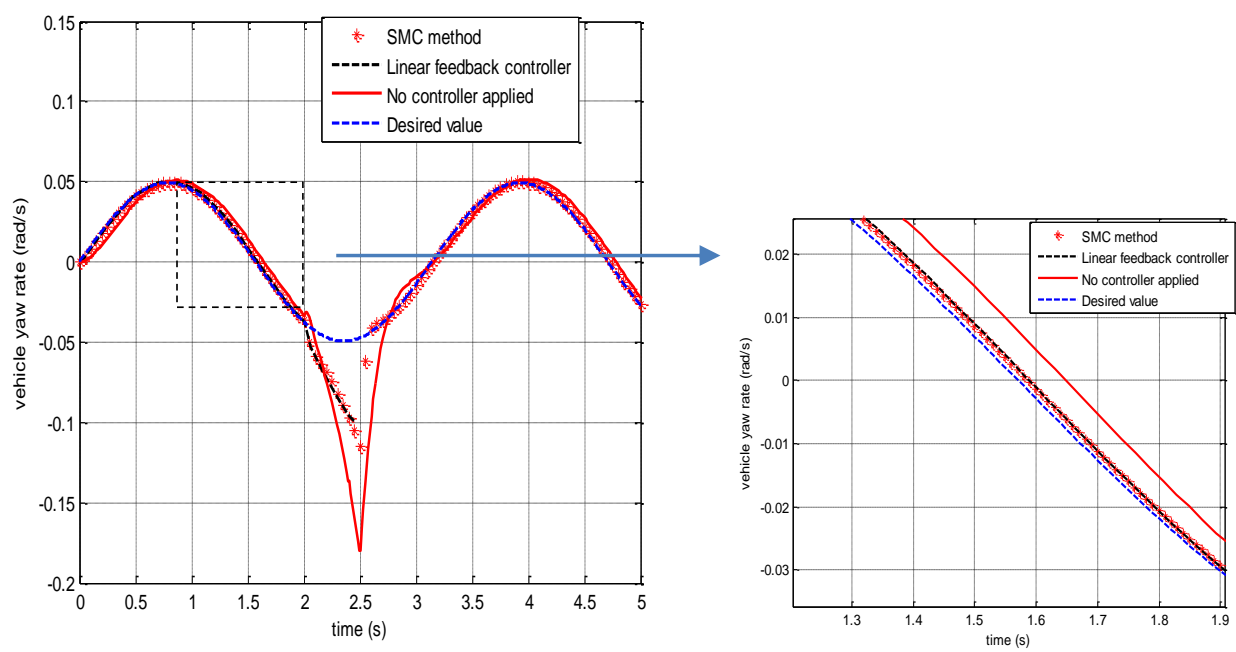

Figure 7. Vehicle controlled yaw rate during the motion of single lane change. (two faulty wheels)

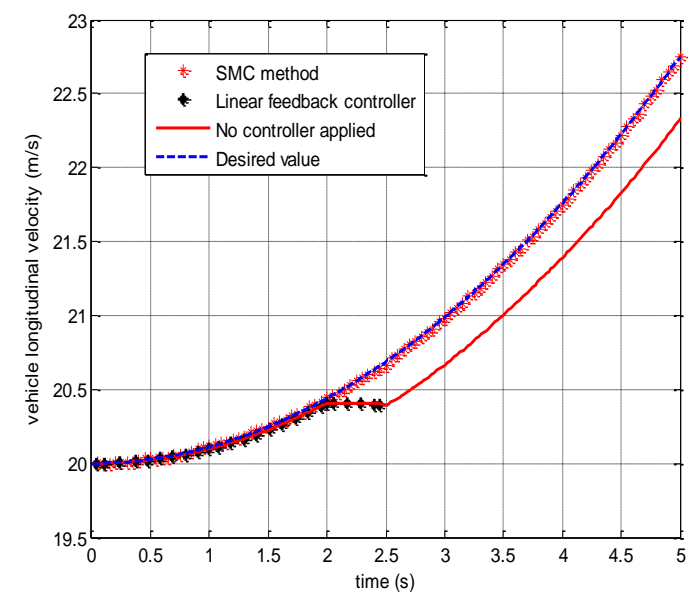

Figure 8 . Vehicle longitudinal velocity during the motion of single lane change. (two faulty wheels)

In the second set of simulations, the vehicle is performing a simple J-turn motion and the input steering angle is shown in Figure 9. Vehicle initial velocity is $15 \mathrm{~m} / \mathrm{s}$ and friction coefficient is 0.9 . The rear right wheel is faulty from 2 seconds to 4 seconds. In this simulation, the steering angle is large and the primary control targets have changed into the yaw rate and vehicle body slip angle. Thus, in theory, control law $v_{2}$ and control law $v_{3}$ should be applied in this simulation. However, when the steering angle is large, there is a strong coupling effect between the control laws $v_{2}$ and $v_{3}$ and both the control performance of the yaw rate and the body slip angle will be negatively affected. Therefore, the simulation results of the application of yaw rate control law $v_{3}$ in SMC alone and the simulation results of the application of both control laws $v_{2}$ and $v_{3}$ in SMC are compared to show this strong coupling effect. In the next section, this problem is solved by grouping the driving control actuators. The detailed explanation of this and the simulation results can be found in the following sections. 
It should be noted that the only application of yaw rate control law $v_{3}$ is briefly called yaw rate SMC and the application of both control laws $v_{2}$ and $v_{3}$ is called combined SMC in the following paragraph.

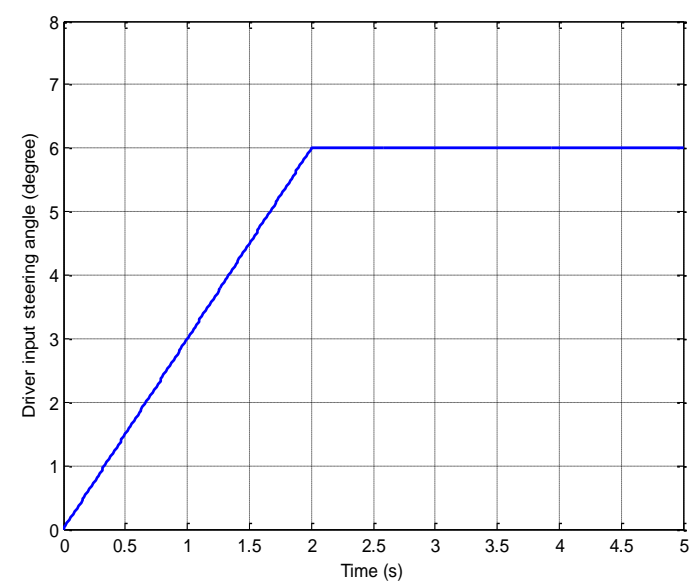

Figure 9. Driver's input steering angle during a J-turn manoeuvre.

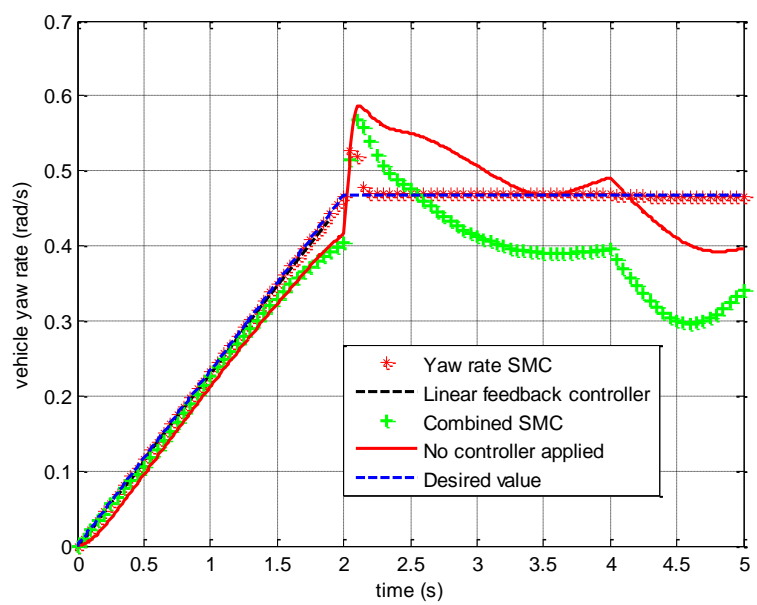

Figure 10. Vehicle controlled yaw rate during a J-turn manoeuvre. (one faulty wheel)

According to Figure 10, the yaw rate SMC method can track the desired yaw rate response perfectly even though the rear right wheel is faulty after 2 seconds. The yaw rate control performance of the combined SMC is compromised due to the coupling effect between control laws $v_{2}$ and $v_{3}$. The linear feedback controller also has a serious error during 2 seconds and the simulation stops at 2 seconds, which is obviously not suitable to the faulttolerant control. In Figure 11, the simulation of the linear feedback control stops due to the wheel fault in 2 seconds. The combined SMC method shows even worse body slip angle response compared with the no controller applied situation and the yaw rate SMC method. Since the combined SMC shows no advantages over the yaw rate SMC, only yaw rate SMC is applied under the large steering angle turning condition in the following simulation. In the next section, the body slip angle performance when the SMC method is applied is improved by grouping the driving actuators. 


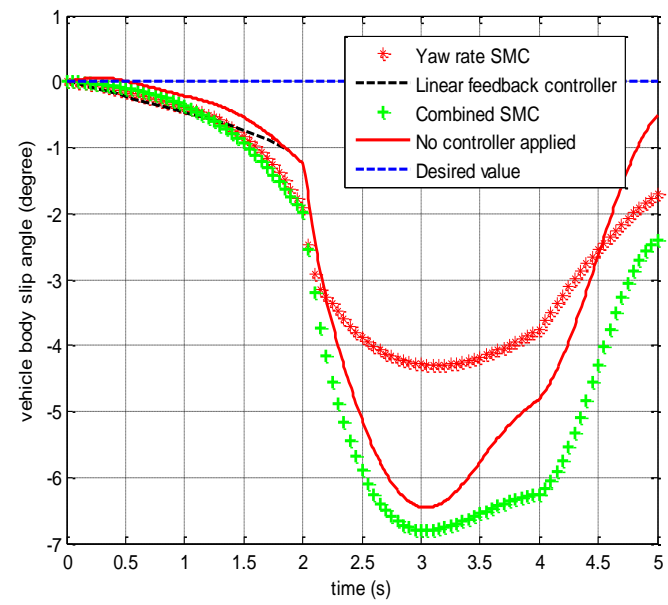

Figure 11. Vehicle body slip angle performance during J-turn manoeuvre. (one faulty wheel)

Figure 13 shows the yaw rate response and Figure 14 shows the body slip angle response when the two rear wheels are assumed to be faulty. From 2 seconds to 4 seconds, the rear right wheel of the electric vehicle is faulty. Moreover, the two rear wheels are faulty from 4 seconds to 5 seconds. The motor control gains of the two rear wheels are shown in Figure 12. In the simulation, the application of the linear feedback controller stops at 2 seconds. The yaw rate SMC method cannot achieve good control of the yaw rate and body slip angle after 4 seconds since all the two rear wheels lost the control. However, the control performance of SMC method is still better than no controller applied condition.

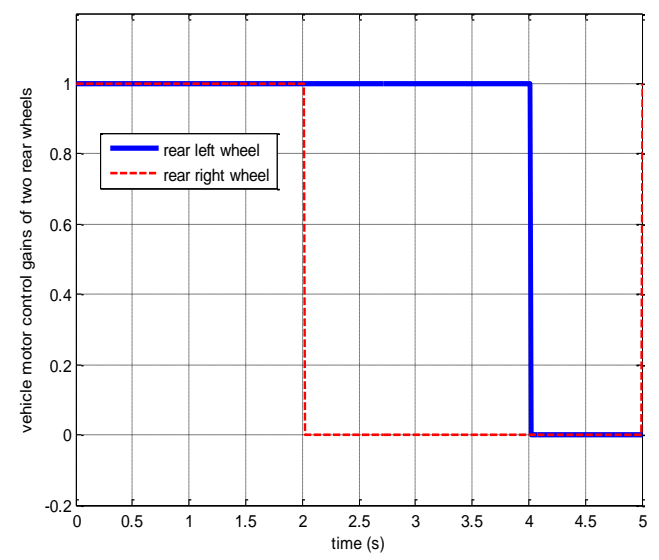

Figure 12. The motor control gains of two rear wheels during J-turn manoeuvre. (two faulty wheels) 

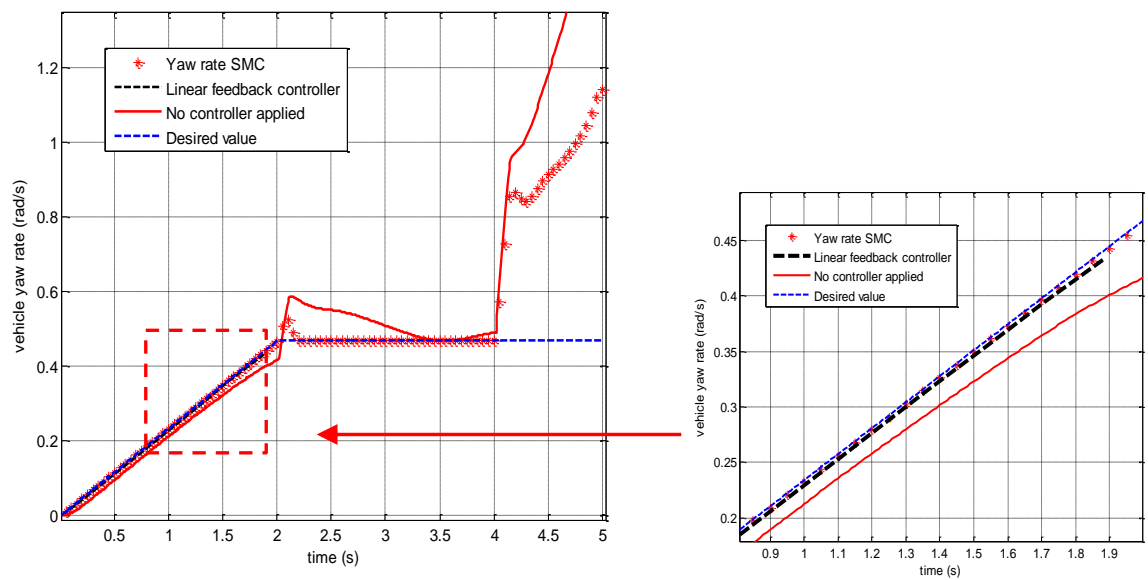

Figure 13. Vehicle controlled yaw rate during a J-turn manoeuvre. (two faulty wheels)

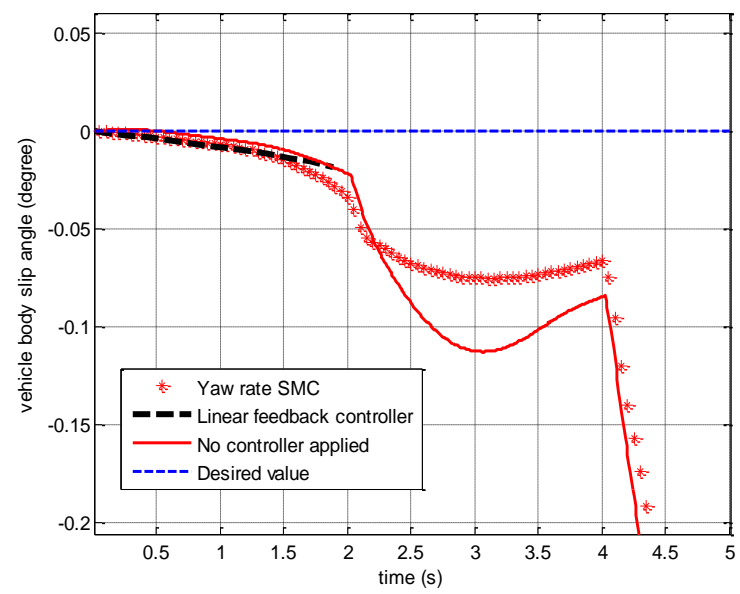

Figure 14. Vehicle body slip angle performance during J-turn manoeuvre. (two faulty wheels)

In this section, the simulation results prove that the proposed SMC controller can achieve better control under normal driving conditions and in a large steering angle J-turn manoeuvre than the linear feedback controller but the proposed SMC method still has some disadvantages. For instance, large sliding gains $K_{1}, K_{2}$ and $K_{3}$ are required in the above two sets of simulations in order to achieve good control performance. These large values will induce the chattering effects caused by frequent switching around the sliding surface. In addition, during the J-turn manoeuvre with a large steering angle, due to the strong coupling effect between the lateral velocity control $v_{2}$ and the yaw rate control $v_{3}$, only the yaw rate control $v_{3}$ is applied, and consequently the body slip angle response is compromised. 


\section{Innovative Actuator-grouping SMC Controller}

In this section, some improved SMC methods are proposed to solve the two disadvantages of the SMC controller mentioned in the above section. Alipour et al. [25] introduced the PISMC method, which included a proportional and integral controller into the SMC:

$$
\begin{gathered}
v_{1}=-v_{y} r-B_{y 1} F_{\text {si-linear }}+\dot{v}_{x r}+K_{p 1}\left(v_{x d}-v_{x}\right)+K_{i 1} \int\left(v_{x d}-v_{x}\right)-K_{1} \operatorname{Sat}\left(S_{1}\right) \\
v_{2}=v_{x} r-B_{y 2} F_{\text {si-linear }}+\dot{v}_{y r}+K_{p 2}\left(v_{y d}-v_{y}\right)+K_{i 2} \int\left(v_{y d}-v_{y}\right)-K_{2} \operatorname{Sat}\left(S_{2}\right) \\
v_{3}=-B_{y 3} F_{\text {si-linear }}+\dot{r}_{r}+K_{p 3}\left(v_{y d}-v_{y}\right)+K_{i 3} \int\left(v_{y d}-v_{y}\right)-K_{3} \operatorname{Sat}\left(S_{3}\right)
\end{gathered}
$$

where $K_{p 1}, K_{i 1}, K_{p 2}, K_{i 2}, K_{p 3}, K_{i 3}$ are determined online by the Levenberg Marquardt algorithm (LMA) algorithm, which aims to minimise the tracking error of the yaw rate or body slip angle. The detailed LMA algorithm can be found in [25]. In this study, the LMA algorithm is further revised by adding a threshold value of the yaw rate error or body slip angle error. This is because a too small yaw rate or body slip angle error will cause the singularity of the matrix and the LMA algorithm will not be accurate. On the other hand, a small yaw rate or body slip angle error means that the SMC method has tracked the desired values perfectly and the PI controller is no longer required.

The threshold value of the yaw rate error is defined as $e_{r 0}$ and the threshold value of the body slip angle is defined as $e_{\beta 0}$. If either the actual yaw rate error $e_{r}$ or the body slip angle error $e_{\beta}$ is larger than its threshold value, the PISMC will be actuated to control the vehicle. Otherwise, the traditional SMC is applied. The detailed structure of this threshold selection method is shown in Figure 15. 


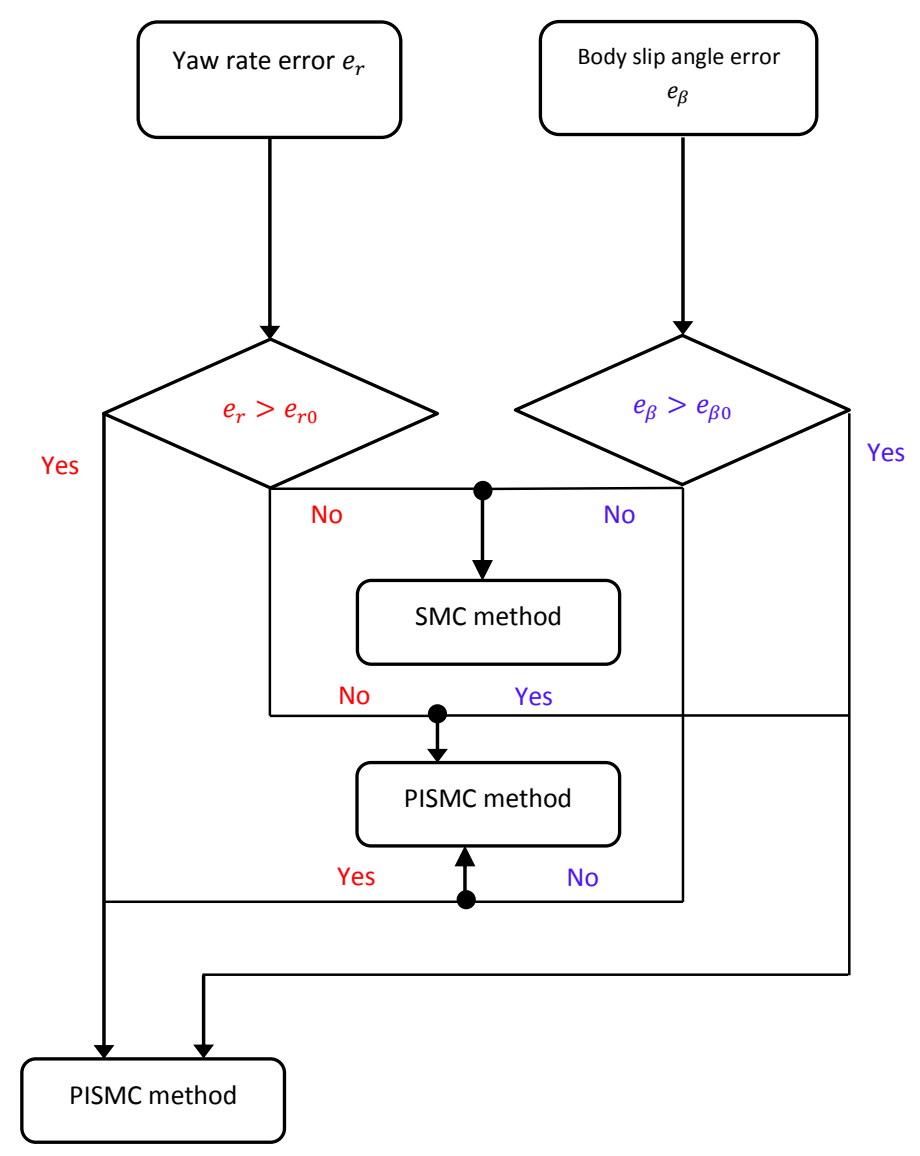

Figure 15. The flow chart of the threshold selection method of PISMC.

In the 4WID vehicle, there are four driving actuators which can be utilised if all the wheels are in the healthy condition. When there is a large steering angle during turning, the control targets are yaw rate and body slip angle. When all the four wheels are used simultaneously to control the yaw rate and body slip angle, there is a strong coupling effect on these two control targets. To solve this problem, the four driving motors are grouped into the class of the two front driving motors and the class of the two rear driving motors. If front wheels are steering wheels, the two front wheels are used to control the body slip angle and the two rear wheels are used to control the yaw rate. Similarly, if the rear wheels are steering wheels, the two rear wheels are used to control the body slip angle and the two front wheels are used to control the yaw rate. This is because only the steering wheel can generate enough vehicle lateral tyre force to control the vehicle body slip angle and all the four wheels can generate enough yaw moment to control the yaw rate. This control law can be considered as the revised SMC controller as follows:

$$
\begin{gathered}
v_{1}=\frac{1}{R_{\omega} m}\left(\cos \delta_{f l} k_{f l} u_{f l}+\cos \delta_{f r} k_{f r} u_{f r}+\cos \delta_{r l} k_{r l} u_{r l}+\cos \delta_{r r} k_{r r} u_{r r}\right)=-v_{y} r- \\
B_{y 1} F_{\text {si-linear }}+\dot{v}_{x r}-K_{1} \operatorname{Sat}\left(S_{1}\right)
\end{gathered}
$$




$$
\begin{array}{r}
v_{2}=\frac{1}{R_{\omega} m}\left(a_{1} \sin \delta_{f l} k_{f l} u_{f l}+a_{2} \sin \delta_{f r} k_{f r} u_{f r}+a_{3} \sin \delta_{r l} k_{r l} u_{r l}+a_{4} \sin \delta_{r r} k_{r r} u_{r r}\right)= \\
v_{x} r-B_{y 2} F_{\text {si-linear }}+\dot{v}_{y r}-K_{2} \operatorname{Sat}\left(S_{2}\right) \\
v_{3}=\frac{1}{R_{\omega} I_{z}}\left(b_{1}\left(l_{f} \sin \delta_{f l}+\frac{b_{f}}{2} \cos \delta_{f l}\right) k_{f l} u_{f l}+b_{2}\left(l_{f} \sin \delta_{f r}-\frac{b_{f}}{2} \cos \delta_{f r}\right) k_{f r} u_{f r}+\right. \\
\left.b_{3}\left(\frac{b_{r}}{2} \cos \delta_{r l}-l_{r} \sin \delta_{r l}\right) k_{r l} u_{r l}+b_{4}\left(-\frac{b_{r}}{2} \cos \delta_{r r}-l_{r} \sin \delta_{r r}\right) k_{r r} u_{r r}\right)= \\
-B_{y 3} F_{s i-l i n e a r}+\dot{r}_{r}-K_{3} \operatorname{Sat}\left(S_{3}\right)
\end{array}
$$

where if the vehicle is front wheel steering, $a_{1}=a_{2}=b_{3}=b_{4}=1$ and $a_{3}=a_{4}=b_{1}=$ $b_{2}=0$. If the vehicle is rear wheel steering, $a_{1}=a_{2}=b_{3}=b_{4}=0$ and $a_{3}=a_{4}=b_{1}=$ $b_{2}=1$.

If there is one faulty wheel among the four wheels, the front left wheel and rear right wheel can be put into a group, and front right wheel and rear left wheel can be put into a group. In this way, we can guarantee there are always two driving wheels being utilised to control the vehicle yaw rate, since one wheel is not enough to control the yaw rate. In equation (38), if the vehicle is front wheel steering and the faulty wheel is the rear left wheel, $a_{2}=b_{1}=b_{4}=$ 1 and $a_{1}=a_{4}=b_{2}=0$. If the vehicle is front wheel steering and the faulty wheel is the rear right wheel, $a_{1}=b_{2}=b_{3}=1$ and $a_{2}=a_{3}=b_{1}=0$. If the vehicle is rear wheel steering and the faulty wheel is the front left wheel, $a_{4}=b_{2}=b_{3}=1$ and $a_{2}=a_{3}=b_{4}=0$. If the vehicle is rear wheel steering and the faulty wheel is the front right wheel, $a_{3}=b_{1}=b_{4}=1$ and $a_{1}=a_{4}=b_{3}=0$.

If the two front wheels or two rear wheels of the vehicle are faulty, the vehicle can still perform the cornering motion. In this way, there are two wheels left to be controlled. In this situation, these two wheels are used to control the yaw rate and the vehicle body slip angle cannot be controlled due to the limited number of driving actuators. Therefore, equation (38) can be represented as follows:

If the two front wheels are faulty:

$$
\begin{array}{r}
v_{3}=\frac{1}{R_{\omega} I_{z}}\left(b_{3}\left(\frac{b_{r}}{2} \cos \delta_{r l}-l_{r} \sin \delta_{r l}\right) k_{r l} u_{r l}+b_{4}\left(-\frac{b_{r}}{2} \cos \delta_{r r}-l_{r} \sin \delta_{r r}\right) k_{r r} u_{r r}\right)= \\
-B_{y 3} F_{\text {si-linear }}+\dot{r}_{r}-K_{3} \operatorname{Sat}\left(S_{3}\right)
\end{array}
$$

where $b_{4}=b_{3}=1$.

If the two rear wheels are faulty:

$$
\begin{array}{r}
v_{3}=\frac{1}{R_{\omega} I_{z}}\left(b_{1}\left(l_{f} \sin \delta_{f l}+\frac{b_{f}}{2} \cos \delta_{f l}\right) k_{f l} u_{f l}+b_{2}\left(l_{f} \sin \delta_{f r}-\frac{b_{f}}{2} \cos \delta_{f r}\right) k_{f r} u_{f r}\right)= \\
-B_{y 3} F_{\text {si-linear }}+\dot{r}_{r}-K_{3} \operatorname{Sat}\left(S_{3}\right)
\end{array}
$$


where $b_{2}=b_{1}=1$.

\section{Simulation Results with Actuator-grouping SMC Controller}

Section $\mathrm{V}$ shows the simulation results which proved that the proposed SMC method can achieve good control performance under normal driving conditions but the control performance of the body slip angle is compromised during turning when there is a large steering angle. In this section, the vehicle under extreme turning conditions is examined where the revised actuator-grouping SMC controller is expected to overcome the compromised control performance of the body slip angle. The simulation performance of PISMC is also tested in order to decrease the sliding mode control gain and decrease the driving control effort. Under extreme turning conditions, the yaw rate and body slip angle are the primary control targets.

Table 1. Parameter values used in simulations. [28][31]

\begin{tabular}{|c|c|c|}
\hline$m$ & Mass & $1298.9 \mathrm{~kg}$ \\
\hline$l_{f}$ & $\begin{array}{c}\text { Distance of c.g. from } \\
\text { the front axle }\end{array}$ & $1 \mathrm{~m}$ \\
\hline$l_{r}$ & $\begin{array}{c}\text { Distance of c.g. from } \\
\text { the rear axle }\end{array}$ & $1.454 \mathrm{~m}$ \\
\hline$b_{f}$ & Front track width & $1.436 \mathrm{~m}$ \\
\hline$b_{r}$ & Rear track width & $1.436 \mathrm{~m}$ \\
\hline$C_{s}$ & $\begin{array}{c}\text { Longitudinal stiffness } \\
\text { of the tyre }\end{array}$ & $\begin{array}{c}50000 \\
\text { N/unit slip } \\
\text { ratio }\end{array}$ \\
\hline$I_{z}$ & $\begin{array}{c}\text { Vehicle moment of } \\
\text { inertial about yaw } \\
\text { axle }\end{array}$ & $1627 \mathrm{kgm}^{2}$ \\
\hline$R_{\omega}$ & Wheel radius & $0.35 \mathrm{~m}$ \\
\hline$I_{\omega}$ & $\begin{array}{l}\text { Wheel moment of } \\
\text { inertial }\end{array}$ & $2.1 \mathrm{kgm}^{2}$ \\
\hline$\varepsilon_{r}$ & $\begin{array}{l}\text { Road adhesion } \\
\text { reduction factor }\end{array}$ & $0.015 \mathrm{~s} / \mathrm{m}$ \\
\hline$C_{\alpha}$ & $\begin{array}{c}\text { Cornering stiffness of } \\
\text { the tyre }\end{array}$ & $\begin{array}{l}30000 \\
\mathrm{~N} / \mathrm{rad}\end{array}$ \\
\hline
\end{tabular}




\begin{tabular}{|c|c|c|}
\hline$t_{m}$ & Mechanical trail & $0.028 \mathrm{~m}$ \\
\hline$t_{p 0}$ & Initial pneumatic trail & $0.05 \mathrm{~m}$ \\
\hline$k$ & $\begin{array}{c}\text { Jack-up moment } \\
\text { coefficient }\end{array}$ & $\begin{array}{c}362 \\
\mathrm{~N} \cdot \mathrm{m} / \mathrm{rad}\end{array}$ \\
\hline$J_{\text {eff }}$ & $\begin{array}{c}\text { Effective rotational } \\
\text { inertia }\end{array}$ & $4 \mathrm{Kg} \cdot \mathrm{m}^{2}$ \\
& $\begin{array}{c}\text { Effective damping } \\
\text { coefficient }\end{array}$ & $\begin{array}{c}88 \\
\mathrm{~N} \cdot \mathrm{m} /(\mathrm{rad} / \mathrm{s})\end{array}$ \\
\hline$b_{\text {eff }}$ & \multicolumn{2}{|c|}{} \\
\end{tabular}

In the first set of simulations, the vehicle is performing the simple J-turn motion and the input steering angle is the same as the value in Figure 9. The initial vehicle velocity is $15 \mathrm{~m} / \mathrm{s}$ and the friction coefficient is 0.9 . We assume the vehicle's rear right wheel is broken between 2 seconds to 4 seconds. First the PISMC method is used in order to attempt to improve the control performance of the traditional SMC method. The yaw rate SMC method is applied as the traditional SMC due to the strong coupling effect between control targets.

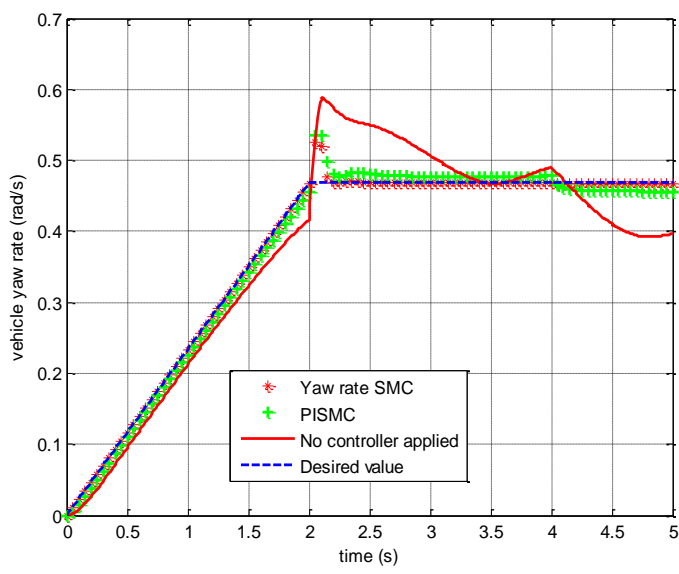

Figure 16. Vehicle yaw rate response during a J-turn manoeuvre when PISMC is applied.

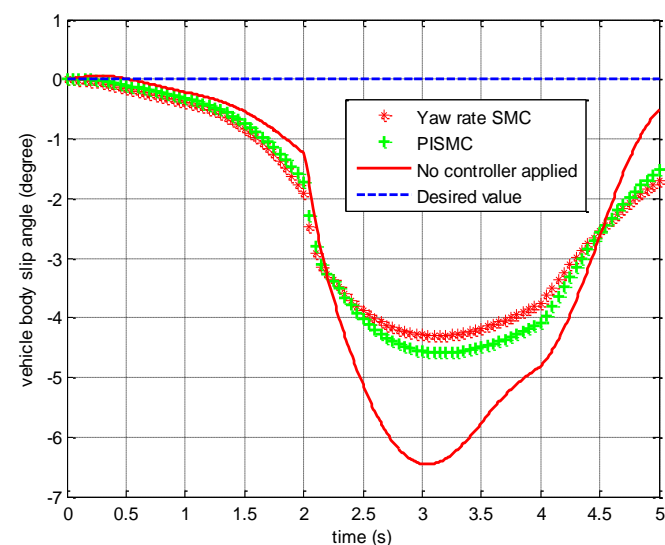


Figure 17 . Vehicle body slip angle response during a J-turn manoeuvre when PISMC is applied

According to Figures 16 and 17, when the PISMC is applied, the stability of the SMC can be improved and the sliding control gain, which is 500 in this simulation, can be decreased. (The default value of sliding mode control gain is 4000 for the traditional SMC method.) The control error of the yaw rate can be compensated for by the PI controller and less control effort is required, as shown in Figures 18 and 19. The improvement in the vehicle body slip angle, however, is still not significant.

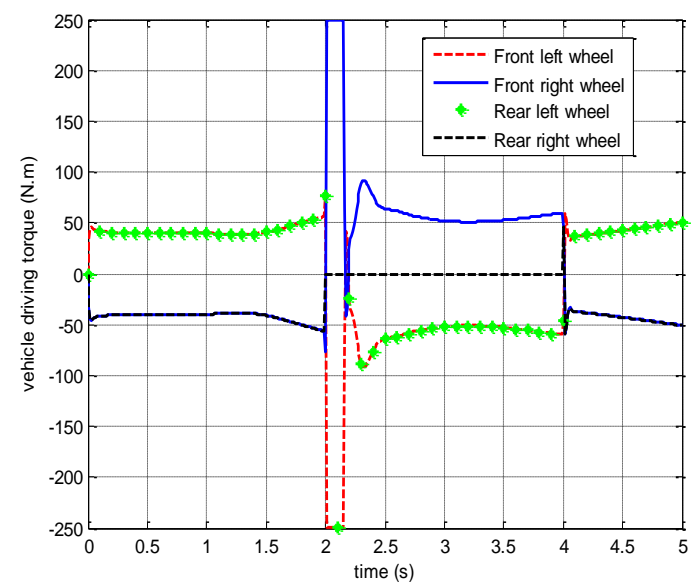

Figure 18. The input driving torque of each individual wheel when traditional SMC method is applied.

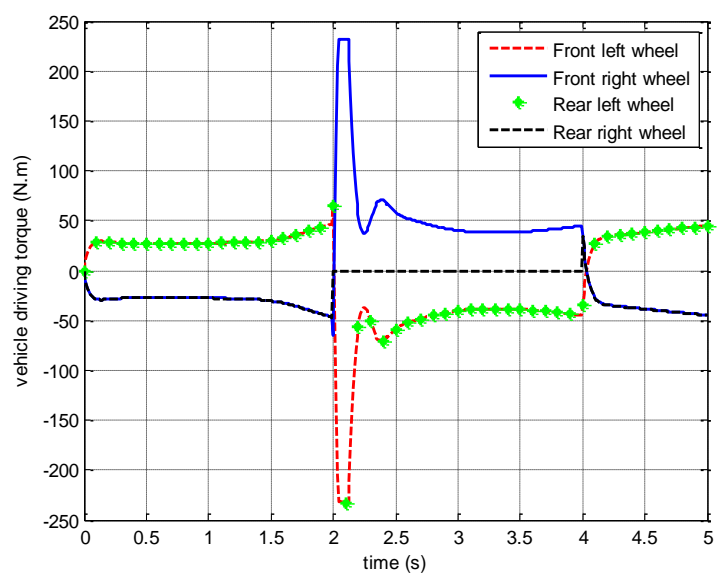

Figure 19. The input driving torque of each individual wheel when PISMC method is applied.

In the next set of simulations, the driver's input steering angle is shown in Figure 20 and all the other conditions remain unchanged. Figures 21 and 22 show the vehicle yaw rate response and body slip angle response when the revised actuator-grouping SMC method is applied. In Figure 21, the yaw rate controlled by the traditional SMC method and revised actuator-grouping SMC method can both achieve the desired yaw rate accurately. According to Figure 22, the body slip angle control performance of the revised actuator-grouping SMC 
method is significantly better than the traditional SMC method and also better than the no controller applied condition. When no controller is applied, the yaw rate control performance is much worse than the controlled methods. The sharp increase of the yaw rate at 2 seconds is mainly because the rear right wheel is faulty and the steering angle is no longer controlled by the driver at this time. In addition, in order to comprehensively analyse the vehicle stability performance, the value of the vehicle body slip angle rate is also introduced and shown in Figure 23. In Figure 23, the actuator-grouping SMC also shows advantage over the traditional SMC.

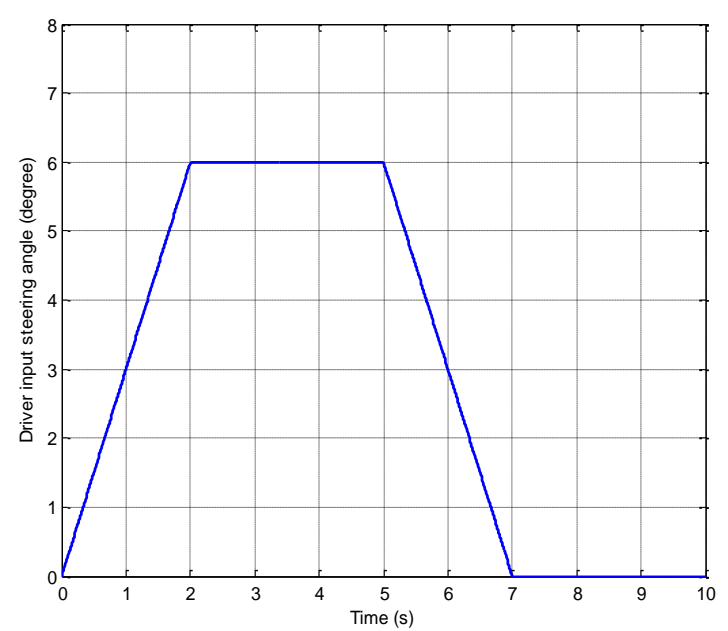

Figure 20. The driver's steering input when the revised actuator-grouping SMC controller is used.

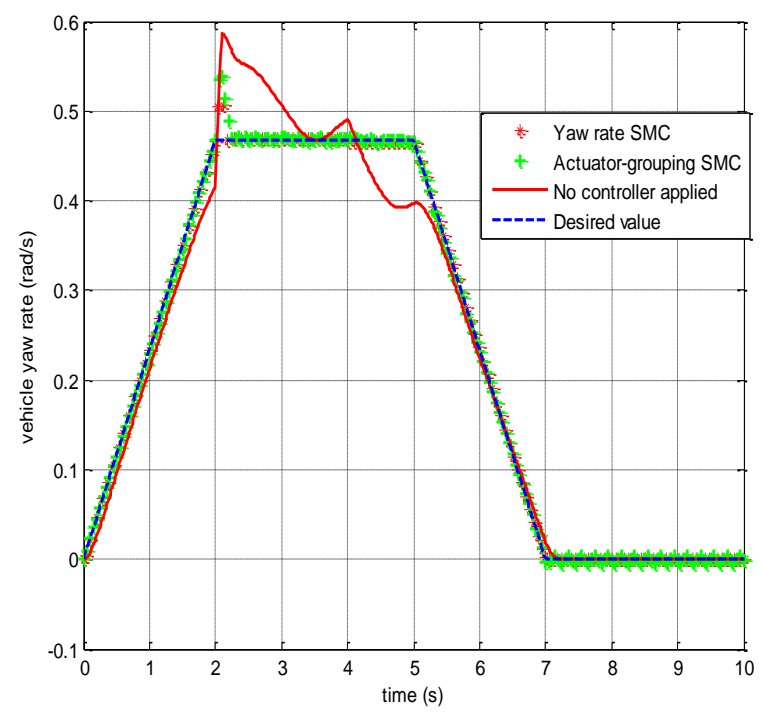

Figure 21. Vehicle yaw rate response when the revised actuator-grouping SMC controller is used. (one faulty wheel) 


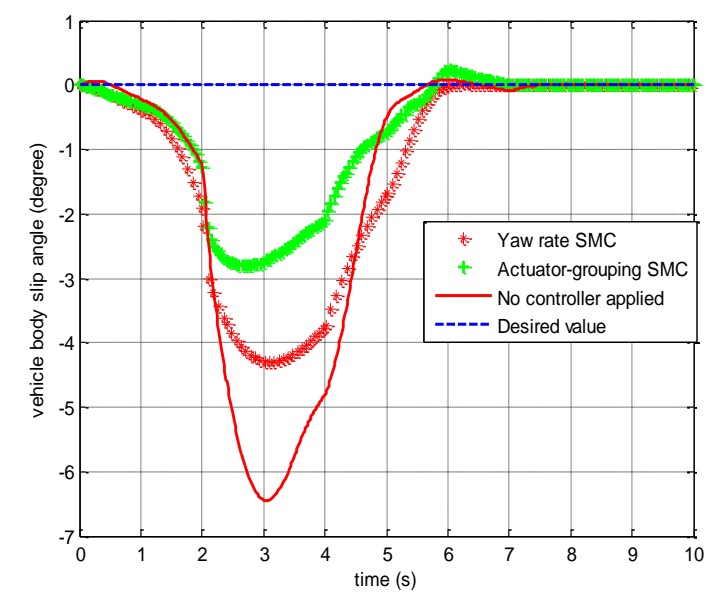

Figure 22. Vehicle body slip angle response when the revised actuator-grouping SMC controller is used. (one faulty wheel)

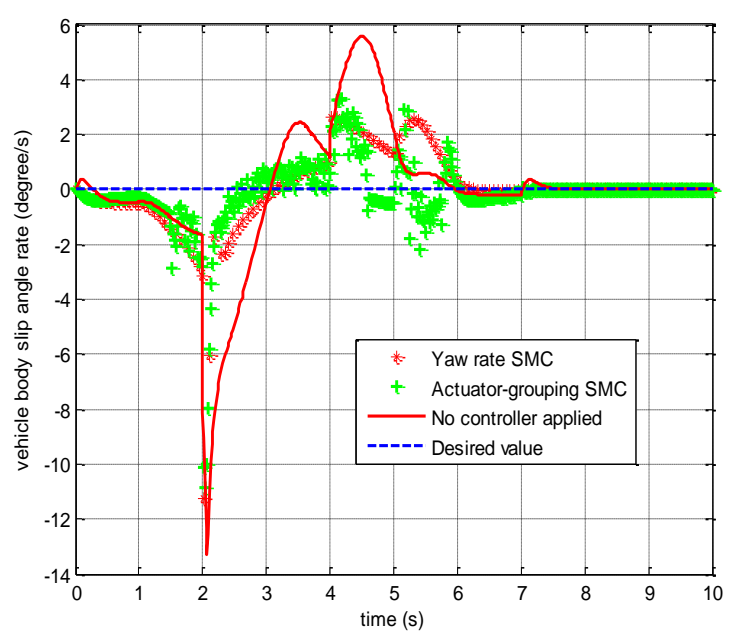

Figure 23. Vehicle body slip angle change rate when the revised actuator-grouping SMC controller is used. (one faulty wheel)

In the above simulations, it is assumed that only the rear right wheel cannot work for between 2 to 4 seconds. In the following simulation, the rear right wheel can be assumed as the faulty wheel from 4 to 6 seconds. After that, the two rear wheels are both assumed to be faulty from 6 to 7 seconds and only the two front wheels can be steered and driven to maintain the vehicle dynamics performance. The motor control gains of the two faulty rear wheels are shown in Figure 24. 


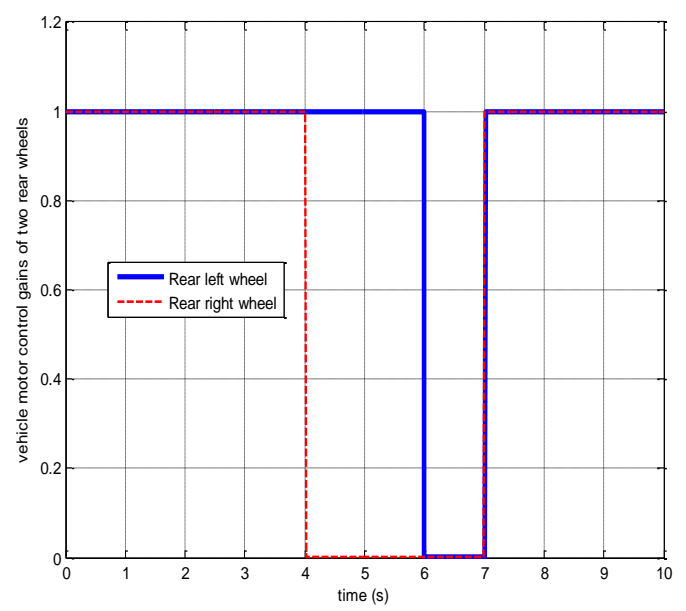

Figure 24. The motor control gains of two rear wheels. (two faulty wheels)

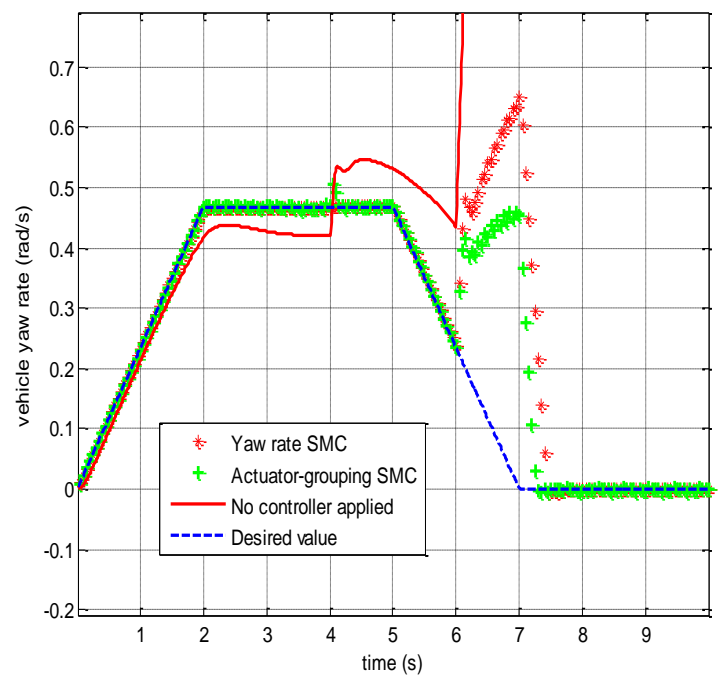

Figure 25. Vehicle yaw rate response when the revised actuator-grouping SMC controller is used. (two faulty wheels)

According to Figure 25, the traditional SMC method and the proposed actuator-grouping SMC method can achieve the desired yaw rate perfectly when only one wheel does not work or no fault happens. When two rear wheels are faulty, the yaw rate responses of two SMC methods increase sharply because the steering angles of two rear wheels are uncontrolled. The revised actuator-grouping SMC shows better yaw rate control performance than the traditional SMC and no controller applied condition. The body slip angle performance in Figure 26 and body slip angle change rate performance in Figure 27 are similar to Figure 22 and Figure 23, respectively and this proves that the proposed actuator-grouping SMC method can significantly improve the body slip angle response even when two rear wheels are faulty. 


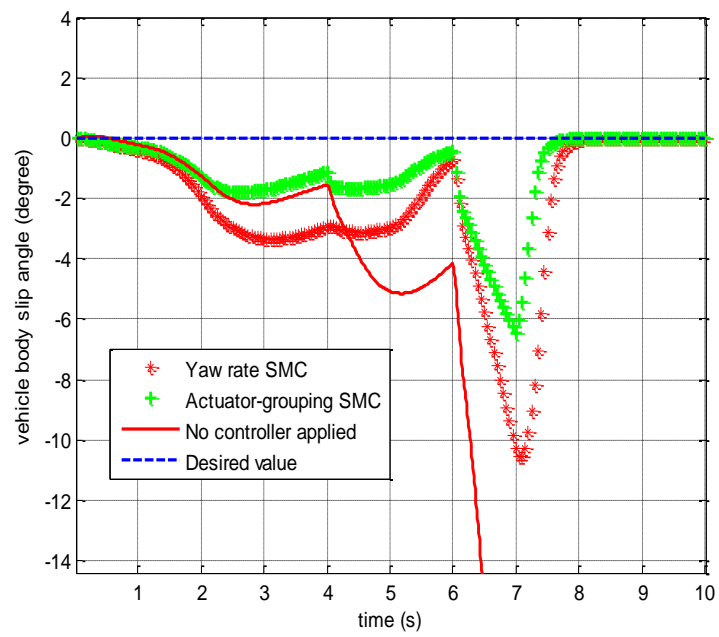

Figure 26. Vehicle body slip angle response when the revised actuator-grouping SMC controller is used. (two faulty wheels)

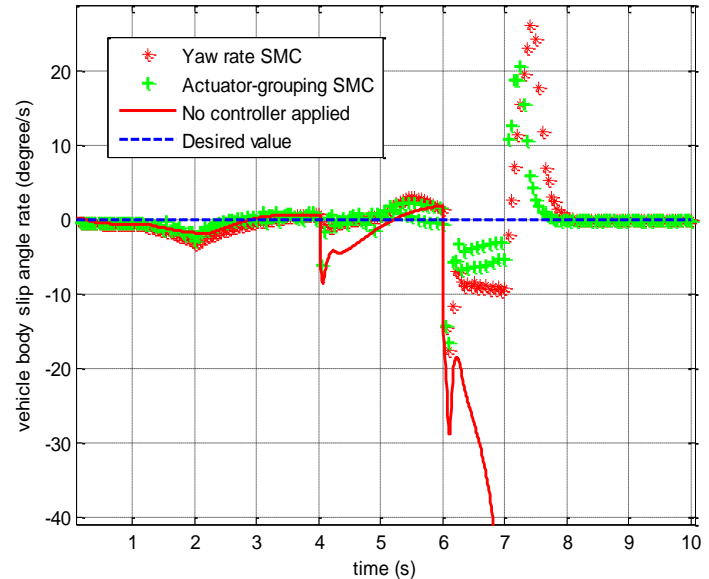

Figure 27. Vehicle body slip angle change rate when the revised actuator-grouping SMC controller is used. (two faulty wheels)

To better present and compare the simulation results of the vehicle yaw rate, body slip angle and longitudinal velocity, the root mean square (RMS) values of overall output response can be presented by the following equation:

$$
R M S=R_{1} \sqrt{\operatorname{mean}\left(v_{x d}-v_{x}\right)^{2}}+R_{2} \sqrt{\operatorname{mean}\left(\beta_{d}-\beta\right)^{2}}+R_{3} \sqrt{\operatorname{mean}\left(r_{d}-r\right)^{2}}
$$

The term mean $(x)$ means the average value of the argument $x$. Each term is corresponding to one specific control target's RMS error value. $R_{1}, R_{2}, R_{3}$ are the scaling factors of each term. The default values of $R_{1}, R_{2}$ and $R_{3}$ are 1 , which represents the equal weighting of each term. If one specific control target is focused, the corresponding scaling factor can be 
increased. It has been suggested that the control targets can be different in different vehicle moving conditions and consequently the values of these scaling factors can be different. Particularly, in the normal driving condition, longitudinal velocity and yaw rate control are focused $\left(R_{1}=1, R_{2}=0, R_{3}=1\right)$. In the vehicle J-turn motion, the vehicle body slip angle and yaw rate control are focused $\left(R_{1}=0, R_{2}=1, R_{3}=1\right)$.

Tables 2 and 3 summarise the RMS values of the body slip angle response and yaw rate response in the second and last set of simulations, which are corresponding to Figures 20-27. These two sets of simulations are all J-turn motions and consequently the scaling factors $R_{1}=0, R_{2}=1, R_{3}=1$. Table 2 and Table 3 suggest that the proposed actuator-grouping SMC has much better overall control performance compared with yaw rate SMC and no controller applied condition in the simulation when only one wheel is faulty or in the simulation when two rear wheels are faulty.

Table 2. RMS values of control targets in the second set of simulations.

\begin{tabular}{|c|c|c|c|c|}
\hline Control method & $\begin{array}{c}\text { Longitudinal } \\
\text { velocity error }\end{array}$ & $\begin{array}{c}\text { Body slip angle } \\
\text { error }\end{array}$ & Yaw rate error & Overall error \\
\hline $\begin{array}{c}\text { No controller } \\
\text { applied }\end{array}$ & 0 & 0.0452 & 0.0346 & 0.0798 \\
\hline Yaw rate SMC & 0 & 0.0350 & 0.0057 & 0.0407 \\
\hline $\begin{array}{c}\text { Actuator-grouping } \\
\text { SMC }\end{array}$ & 0 & 0.0215 & 0.0084 & 0.0299 \\
\hline
\end{tabular}

Table 3. RMS values of control targets in the last set of simulations.

\begin{tabular}{|c|c|c|c|c|}
\hline Control method & $\begin{array}{c}\text { Longitudinal } \\
\text { velocity error }\end{array}$ & $\begin{array}{c}\text { Body slip angle } \\
\text { error }\end{array}$ & Yaw rate error & Overall error \\
\hline $\begin{array}{c}\text { No controller } \\
\text { applied }\end{array}$ & 0 & 0.1333 & 0.3906 & 0.5239 \\
\hline Yaw rate SMC & 0 & 0.0580 & 0.1609 & 0.2189 \\
\hline $\begin{array}{c}\text { Actuator-grouping } \\
\text { SMC }\end{array}$ & 0 & 0.0324 & 0.1088 & 0.1412 \\
\hline
\end{tabular}

\section{Conclusions}

This study first suggests some modifications to the traditional SMC method to achieve faulttolerant control of a 4WIS-4WID electric vehicle. The steering geometry must be re-arranged according to the location of the faulty wheels. In addition, three SMC control laws (longitudinal velocity control, lateral velocity control and yaw rate control) can be selected freely based on the specific vehicle motion scenarios. 
In Section V, these modifications on the SMC method are compared with the linear feedback control method and the major findings can be summarised as follows:

1) Under normal driving conditions, the SMC method can achieve the desired yaw rate accurately when one or two wheels are faulty. The linear feedback method always has serious error and is not suitable for the fault tolerant control. In addition, the SMC method has better control performance over the longitudinal velocity compared with the linear feedback method due to the application of virtual control law $v_{1}$.

2) In the scenario of large steering angle turning, the SMC method can achieve the desired yaw rate when one of the front wheels or two front wheels are faulty, while the simulation of the linear feedback control method stops when the wheel fault happens. This proves the robustness of the SMC method. However, the vehicle body slip angle performance is compromised due to the coupling effect between different control targets.

To solve this problem, the driving actuators can be grouped and each group of actuators can be used to achieve the specific control target. This avoids the strong coupling effect between the individual control targets. The simulation in Section VII still uses a large steering angle turning scenario to test the control performance of this revised actuator-grouping SMC method. The simulation results prove that both the body slip angle and the body slip angle rate are significantly improved compared with the traditional SMC method when one or two wheels are faulty.

In the future, an actual experimental vehicle test platform will need to be built to test the control performance of the proposed modified fault-tolerant SMC controller.

\section{Acknowledgement}

This research was supported under Australian Research Council's Discovery Projects funding scheme (project number DP140100303). The authors wish to gratefully acknowledge the help of Dr. Madeleine Strong Cincotta in the final language editing of this paper.

\section{References}

[1] Yamakawa, J., Kojima, A., and Watanabe, K., "A Method of Torque Control for Independent Wheel Drive Vehicles on Rough Terrain,” J. Terramech., vol.44, no.5, pp.371$381,2007$.

[2] Shino, M., and Nagai, M., "Independent Wheel Torque Control of Small Scale Electric Vehicle for Handling and Stability Improvement," JSAE Rev., vol.24, no.4, pp.449-456, 2003. 
[3] Piyabongkarn, D., Rajamani, R., and Lew, J.Y., "Active Driveline Torque Management Systems - Individual Wheel Torque Control for Active Automotive Safety Applications," IEEE Control Syst. Mag., vol.30, no.4, pp.86-102, 2010.

[4] Wang, J., and Hsieh, M.F., "Vehicle Yaw-Inertial- and Mass-Independent Adaptive Steering Control," Proc. Inst. Mech. Eng., Part D (J. Automob. Eng.), vol.233, no.9, pp.11011108, 2009.

[5] Wang, R., and Wang, J., "Fault-tolerant control for electric ground vehicles with independently-actuated in-wheel motors", Journal of Dynamic Systems, Measurement, and Control, vol.134,pp.021014_1-10, 2012.

[6] Fujimoto, H., Saito, T., and Noguchi, T., "Motion stabilization control of electric vehicle under snowy conditions based on yaw-moment observer", in Proceedings of IEEE international workshop on advanced motion control, pp.35-40, Kawasaki, Japan, 2004.

[7] Sakai, S., and Hori, Y., "Advantage of electric motor for anti-skid control of electric vehicle”, European Power Electronics Journal, vol.11, pp.26-32, 2001.

[8] Saito, T., Fujimoto, H., and Noguchi, T., "Yaw-moment stabilization control of small electric vehicle", in Proceedings of the IEEJ technical meeting on industrial instrumentation and control, pp.83-88, Tokyo, Japan, 2002.

[9] Yin, D., and Hori, Y., "A new approach to traction control of EV based on maximum effective torque estimation", in Proceedings of the $34^{\text {th }}$ annual conference of the IEEE industrial electronics society, pp.2764-2769, Florida, USA, 2008.

[10] Hu, J., Yin, D., and Hori, Y., "Fault-tolerant traction control of electric vehicles", Control Engineering Practice, vol.19, pp.204-213, 2011.

[11] Niemann, H., and Stoustrup, J., "Passive fault tolerant control of a double inverted pendulum - a case study", Control Engineering Practise, vol.13, no.8, pp.1047-1059, 2005.

[12] Espinoza, D.R., and Campos-Delgado, D.U., "Active fault tolerant scheme for variable speed drives under actuator and sensor faults", in Proc. IEEE Int.Conf.ControlAppl., pp.474479, San Antonio, TX, USA, 2008.

[13] Zhou, K., "A new controller architecture for high performance, robust, and fault tolerant control", IEEE Trans. Autom. Control, vol.46, no.10, pp.1613-1618, 2001.

[14] Raisemche, A., Boukhnifer, M., Larouci, C., and Diallo, D., "Two active fault-tolerant control schemes of induction-motor drive in EV or HEV", IEEE Transactions on Vehicular Technology, vol.63, no.1, 2014.

[15] Chamseddine, A., and Noura, H., 'Control and Sensor Fault Tolerance of Vehicle Active Suspension," IEEE Trans. Control Syst. Technol., vol.16, no.3, pp.416-433, 2008. 
[16] Jayabalan, R., and Fahimi, B., 'Monitoring and Fault Diagnosis of Multi-converter Systems in Hybrid Electric Vehicles,' IEEE Trans. Veh. Technol., vol.55, no.5, pp.1475-1484, 2006.

[17] Oudghiri, M., Chadli, M., Hajjaji, A., 'Robust Observer-based Fault-tolerant Control for Vehicle Lateral Dynamics,' Int. J. Veh. Des., vol.48, no.3/4, pp.173-189, 2008.

[18] Wallmark, O., Harnefors, L., and Carlson, O., 'Control Algorithms for a Fault-tolerant PMSM Drive', IEEE Trans. Ind. Electron. Control Instrum., vol.54, no.4, pp.1973-1980, 2007.

[19] Muenchhof, M., Beck, M., and Isermann, R., 'Fault-tolerant Actuators and Drives Structures, Fault Detection Principles and Applications," Annu. Rev. Control, vol.33, no.2, pp.136-148, 2009.

[20] Wang, R., and Wang, J., 'Fault-tolerant Control with Active Fault Diagnosis for Fourwheel Independently Driven Electric Ground Vehicles', IEEE Transactions on Vehicular Technology, vol.60, no.9, pp.4276-4287, 2011.

[21] H. Shimizu, J. Harada, and L. Chan, 'Development of a high performance electric vehicle', Proceedings of the 1996 IEEE IECON 22 $2^{\text {nd }}$ International Conference, pp.14-19, 1996.

[22] C. Wenbo, L. Yugong, H. Yunwu, L. Keqiang, 'Rule-based traction system failure control of distributed electric drive vehicle', Journal of Mechanical Engineering, vol.48, no.10, pp.90-95, 2012.

[23] X. Xin, H. Zheng, H. Xu and G. Qin, 'Control strategies for four in-wheel driven electric vehicles when motor drive system fail', 2014 American Control Conference (ACC), pp.885890, Portland, Oregon, USA, 2014.

[24] Wang, R., and Wang, J., 'Passive Actuator Fault-tolerant Control for a Class of Overactuated Nonlinear Systems and Applications to Electric Vehicles', IEEE Transactions on Vehicular Technology, pp.972-985, vol.62, no.3, 2013.

[25] Alipour, H., Sharifian, M. B. B., and Sabahi, M., 'A Modified Integral Sliding Mode Control to Lateral Stabilisation of 4-Wheel Independent Drive Electric Vehicle', Vehicle System Dynamics, vol.52, no.12, pp.1584-1606, 2014.

[26] Song, P., Tomizuka, M., and Zong, C., 'A novel integrated chassis controller for full drive-by-wire vehicles', Vehicle System Dynamics, vol.53, no.2, pp.215-236, 2015.

[27] Li, B.Y., Li, W.H., Kennedy, O., and Du, H.P., "The dynamics analysis of an omnidirectional vehicle," International Journal of Automotive Technology, vol.15, no.3, pp.387398, 2014.

[28] Boada, B., Boada, M. and Díaz, V., "Fuzzy-logic applied to yaw moment control for vehicle stability," Vehicle System Dynamics, vol.43, pp.753-770, 2005. 
[29] Dugoff, H., Fancher, P.S. and Segel, L., "An analysis of tire traction properties and their influence on vehicle dynamic performance”, SAE 700377, pp. 1219-1243, 1970.

[30] Hsu, Y.J., Laws, S.M., and Gerdes, J.C., "Estimation of tire slip angle and friction limits using steering torque", IEEE Transactions on Control System Technology, vol.18, no.4, pp.896-907, 2010.

[31] Ahn, C., Peng, H., and Tseng, H.E., "Robust estimation of road friction coefficient using lateral and longitudinal vehicle dynamics", Vehicle System Dynamics, vol.50, no.6, pp.961985, 2012.

[32] Li, B., Du, H., Li, W. and Zhang, Y., "Side-slip angle estimation based lateral dynamics control for omni-directional vehicles with optimal steering angle and traction/brake torque distribution", Mechatronics, http://dx.doi.org/10.1016/j.mechatronics.2014.12.001, 2014.

[33] Lam, T., Qian, H. and Xu, Y., "Omnidirectional steering interface and control for a fourwheel independent steering vehicle," IEEE/ASME Transactions on Mechatronics, vol.15, pp.329-338, 2010.

[34] Wang, J., Alexander, L., and Rajamani, R., "Friction estimation on high-way vehicles using longitudinal measurements", ASME J. Dyn. Syst.,Meas. Control, vol.126, no.2, pp.265$275,2004$.

[35] Rajamani, R., Phanomchoeng,G., Piyabongkarn, D., and Lew, J.Y., "Algorithms for real-time estimation of individual wheel tire-road friction coefficients", IEEE/ASME Transactions on Mechatronics, vol.17, no.6, pp.1183-1195, 2012.

[36] Li, B., Du, H., and Li, W., "Comparative study of vehicle tyre-road friction coefficient estimation with a novel cost-effective method", Vehicle System Dynamics, vol.52, no.8, pp.1066-1098, 2014.

[37] Li, L., Song, J., Kong, L., and Huang, Q., "Vehicle velocity estimation for real-time dynamic stability control", International Journal of Automotive Technology, vol.10, no.6, pp.675-685, 2009.

[38] Li, B., Du, H., and Li, W., "A novel method for side slip angle estimation of omnidirectional vehicles," SAE Int. J. Passeng. Cars - Electron. Electr. Syst., vol.7, no.2, doi:10.4271/2014-01-0303, 2014.

[39] de Castro, R., Tanelli, M., Araújo, R.E., and Savaresi, S.M., "Design of safety-oriented control allocation strategies for overactuated electric vehicles", Vehicle System Dynamics, vol.52, no.8, pp.1017-1046, 2014. 